\title{
Drug-Using Female Sex Workers and HIV Risk: A Systematic Review of the Global Literature
}

\author{
Yeon Jung $\mathrm{Yu}^{1} \&$ Xiaoming $\mathrm{Li}^{2}$ \\ ${ }^{1}$ Department of Anthropology, Western Washington University, Bellingham, WA, USA \\ ${ }^{2}$ Arnold School of Public Health, University of South Carolina, Columbia, SC, USA \\ Correspondence: Yeon Jung Yu, Department of Anthropology, Western Washington University, Bellingham, WA, \\ USA. Tel: 1-360-650-2182. E-mail: Yeon.Yu@wwu.edu
}

Received: January 26, 2018 Accepted: March 5, 2018 Online Published: March 20, 2018

doi:10.5539/gjhs.v10n5p1 URL: https://doi.org/10.5539/gjhs.v10n5p1

\begin{abstract}
This review examines the global literature concerning HIV risk among drug-using female sex workers (DU-FSWs). In the context of HIV prevention, the possible synergetic effects of sexual risk and drug-related risk merit a systematic review to get a better understanding of such effects among this highly vulnerable population. In particular, we look at research on the association between drug use and HIV risk among female sex workers (FSWs) in terms of multiple indicators such as HIV infection, needle sharing, and unprotected sex.

The current review, through synthesizing the findings from 41 studies conducted in multiple nations, reveals a complex picture of HIV risk for DU-FSWs across diverse societies. Research findings are mixed but tend to show that drug-related and sex-related risk behaviors accelerated the risk of HIV/STI among DU-FSWs, underscoring considerable vulnerabilities. However, findings about the level of the association and significance, as well as the mechanisms of HIV transmission, are inconsistent among various empirical studies. The variations in findings may be attributed to the specificities of diverse social contexts, various characteristics of the study samples, and different measurements in different studies. The mixed findings point to the need for more empirical studies targeting DU-FSWs to understand how drug use and sexual risk interactively affect this population differently in different social contexts. Future research should focus on multiple-level risk/preventive factors, assess the overlap between drug-using networks and sexual networks, and identify the synergetic dynamics between drug use and sex work. Development of conceptual frameworks and methodological innovations are also needed.
\end{abstract}

Keywords: drug use, female sex workers (FSWs), HIV/STIs, needle sharing, unprotected sex

\section{Introduction}

This review examines the global literature on HIV/STI risk among drug-using female sex workers (DU-FSWs). We chose a broad definition of female sex workers (FSWs); this includes any woman who provides sexual services for financial compensation including goods or services (Baral et al., 2013). As a result, the target population of this review includes both professional sex workers and women engaging in transactional sex or survival sex. While professional sex workers may be different from women performing survival sex in many aspects of their lives, it is difficult to practically draw a clear line between the two activities, and the health ramifications of the two activities are likely to be similar. Among DU-FSWs, we include both FSWs who use injection drugs (IDU-FSWs) and FSWs who use non-injection drugs (NIDU-FSWs). Alcohol and tobacco users were excluded, although alcohol abuse is common among FSWs (Medhi et al., 2012; Q. Li, X. Li, \& Stanton 2010; Malta et al., 2008; Inciardi, Surratt, \& Kurtz, 2006).

Three routes of HIV transmissions are: (1) blood and blood products; (2) intimate sexual contact (mucosal contact including penile-vaginal contact, penile-anal contact, and by fellatio), or (3) vertical transmission (mother-to-child) during pregnancy, delivery, or breast-feeding (CHLP 2015). Numerous studies indicate that having an STI magnifies the risk of acquiring HIV by anything from two to eight times or more (Boily et al., 2001).

FSWs are a population at high risk for HIV and other sexually transmitted infections (STIs) (Chen et al., 2012; Yu, 2013) and DU-FSWs may be at even greater risk for HIV infection. FSWs who use drugs are at particularly high risk for STIs, as they can be infected with HIV/STIs via their drug use behavior (e.g., needle sharing) as well as through unprotected sex, and they may transmit HIV/STI to others (MAP, 2005; de Graaf, 1995). The close 
association between drug use and the sex trade has been known for many decades (Silbert, Pines, \& Lynch, 1982; Gossop et al., 1994). Previous studies have shown that a significant portion of FSWs were injection drug users (IDUs) globally (Konings, 1996); commercial sex has been significantly correlated with drug abuse in Wuhan, China $(p<0.01$, OR=11.47) (Wei et al., 2004); hard-drug use among FSWs in Durban, South Africa, has been shown to be very common (41\%) (Leggett, 2001); a study showed that 83\% of FSWs in Amsterdam were IDUs (van den Hoek et al., 1988). Injection drug use was inextricably linked to commercial sex (Tyndall et al., 2002). For example, in various locations in the Russian Federation, between 15\% and 66\% of female IDUs were involved in sex work (Platt et al., 2005; Dehne \& Kobyshcha, 2000); over half of female IDUs in China (52-98\%) were involved in sex work (Yang et al., 2005; Gu et al., 2008); and two-thirds of female drug users in Manipur, India, reported exchanging sex for money or drugs (Panda et al., 2001).

Since the 1990s, the high-risk status of DU-FSWs has drawn attention from those involved in both prevention and research efforts. Researchers have increasingly paid attention to DU-FSWs because of the potentially enhanced effects of sexual risks and drug use behaviors (Baral et al., 2013; Malta et al., 2008; Tang et al., 2014; Loza et al., 2010; Lau et al., 2007; Strathdee et al., 2001). The overlap between illicit drug use and sex work has been identified as an important factor responsible for HIV prevalence among FSWs (Medhi et al., 2012), which would indicate double risk for DU-FSWs. However, most researchers only focused on either sexual risks or drug use behavior among FSWs, and few have examined HIV risk among injection and non-injection drug-using FSWs (Tang et al., 2014).

There are several principal explanations for the close linkage between drug use and sex work. First, drug users may begin (and continue) to engage in sex work to support their drug addictions. Since illicit drugs are usually expensive, DU-FSWs often face great economic pressure to support their drug use (Malta et al., 2008; Choi, Cheung, \& Chen, 2006). Many female drug users resort to sex work in order to maintain their drug use, either obtaining drugs from their sexual partners or using the money from sex work to purchase drugs (Medhi et al., 2012; Panda et al., 2001). Second, FSWs may use drugs as a maladaptive means of coping with stress associated with the sex trade (Leggett, 2001; Parry et al., 2009). Third, forceful and/or violent sex partners or clients may coerce FSWs into drug use. Finally, drug use may be consistent with some subcultures of FSWs (e.g., deviant behaviors). This overlap of injection drug use and the sex trade suggests a possible synergy between the two activities in increasing the risk for HIV infection and transmission.

The level of overlap between drug use and the sex trade, however, varied significantly across subpopulations of drug-using women. For example, crack-smoking women (18-29 years of age) in inner-city neighborhoods in New York, Miami, and San Francisco, were 14 times more likely to sell sex compared with non-crack-smoking women (Edlin et al., 1994). Among a group of young women in Miami, Florida, crack use was associated with increased sexual activity, multiple sex partners, and sex work: $89 \%$ of crack-using women engaged in sexual activity; $74 \%$ of them had two or more partners, and $63 \%$ of the women reported sex work (for money or drugs) in the 30 days before the study (Weatherby et al., 1992). IDU-FSWs in Sichuan, China, were at higher risk for HIV infection than female IDUs, as syringe-sharing behaviors (borrowing, lending, injecting with pre-filled syringes) were higher among IDU-FSWs (45.3\%) than female IDUs (37.2\%) (Lau et al., 2008).

A few previous reviews of FSWs and/or drug use have shed light on the great HIV/STI risk among DU-FSWs. A comprehensive review of peer-reviewed articles and gray literature on sex work in Central Asia (Uzbekistan, Tajikistan, Turkmenistan, Kazakhstan, Kyrgyzstan, Mongolia, and Afghanistan) found that there were increasing data directly linking HIV among FSWs to injection drug use (the occurrence of HIV was up to 20 times higher among IDU-FSWs) (Baral et al., 2013). Similarly, a systematic literature review of HIV/STI prevalence data for FSWs in China suggested that IDU-FSWs might be at greater risk for HIV infection, with $12-49 \%$ found to be HIV positive compared with less than 1\% HIV infection rate among FSWs nationwide (Poon et al., 2011). A review on drug-using women within a global framework showed that such women were at particularly high risk not only for HIV but also for other STIs, such as HBV and HCV (Miller \& Neaigus, 2001).

To date, the relationship between drug use and HIV risk among FSWs has been examined in a diverse but limited number of societies, including both high-income countries and low- and mid-income countries. Although existing studies have shed light on the considerable HIV-related risk among DU-FSWs in the past two decades, no systematic review of global literature has focused on DU-FSWs. Thus, we conducted a systematic review by examining the existing global literature on HIV risks among DU-FSWs. In the context of HIV prevention, the synergetic effect of sexual risk and drug-related risk merits such a review to synthesize the existing empirical studies on DU-FSWs at increased risk of exposure to HIV/STIs.

There are three main objectives in the current review: First, to review the empirical evidence on the association 
between drug use and HIV risk among FSWs across different societies. Second, to examine the potential mechanisms through which drug use affects HIV risk among DU-FSWs. Third, to identify the knowledge gap in the current literature and provide recommendations for further research and interventions in the field.

\section{Methods}

\subsection{Inclusion Criteria}

We identified studies that are: (1) peer-reviewed and published in English-language journals prior to November 2014; (2) empirical studies using either qualitative or quantitative methodology; (3) focused on drug-using (either injection or non-injection drugs) with FSWs as the primary target population of the studies; (4) engaged in assessing the association between drug use and HIV-related risks (e.g., prevalence, behaviors) among FSWs.

\subsection{Data Sources}

The literature search was conducted in November 2014, primarily using four electronic bibliographic databases: CINAHL, PsycINFO, PubMed/Medline, and Web of Science. We generated a master list of search terms and tailored search queries to each electronic database. The search terms included FSWs (alternative search terms were: female prostitutes, commercial sex workers, sex exchange, sex trade, survival sex), drug use, and HIV. All citations were imported into EndNote X 7.0 for data management. The search of these four databases resulted in 2,768 citations in total. After deleting duplications, 1,995 citations remained in the EndNote data set for further screening.

\subsection{Screening}

Citations were screened using a three-step process that included a title (and keyword) review, an abstract review, and an article review. At the title review stage, all unduplicated records $[\mathrm{n}=1,995]$ were initially screened to exclude citations that clearly did not provide empirical data for DU-FSWs and HIV/STI risks. As a result, we excluded 1,386 articles: 104 irrelevant citations, 50 non-empirical studies, and 1,232 articles that focused on only one or two of our three primary topics of interest (i.e., sex work, drug use, HIV risk). At the abstract review stage, we excluded 499 of the remaining 609 articles, primarily because they did not examine the relationships between drug use and HIV prevalence or HIV-related behaviors among FSWs. At the article review stage, we excluded 70 of the remaining 110 articles because 53 did not provide data about the relationship between drug use and HIV-related risks, 11 focused on prevention/intervention projects, and the remaining six focused on only one or two of our three primary topics. The three-step screening process left 40 peer-reviewed articles. The references of these 40 eligible articles were then hand-searched with one additional article being identified. The search resulted in 41 eligible articles reporting data from a total of 39 studies for further analysis. Figure 1 illustrates the screening process for each step and presents the reasons for exclusion.

\subsection{Data Abstraction}

We developed structural data abstraction forms to retrieve study characteristics and major findings from each of the 41 reviewed articles. We then created a table to display and categorize the study characteristics (e.g., study location, study sample and size, year of data collection, age of study sample, study design) for each of the reviewed articles. We also created a table that displays the health behaviors and HIV risks (i.e., drug use patterns, HIV prevalence, other STI rates, drug-related risk behaviors, sex-related risk behaviors) for the data we extracted. 


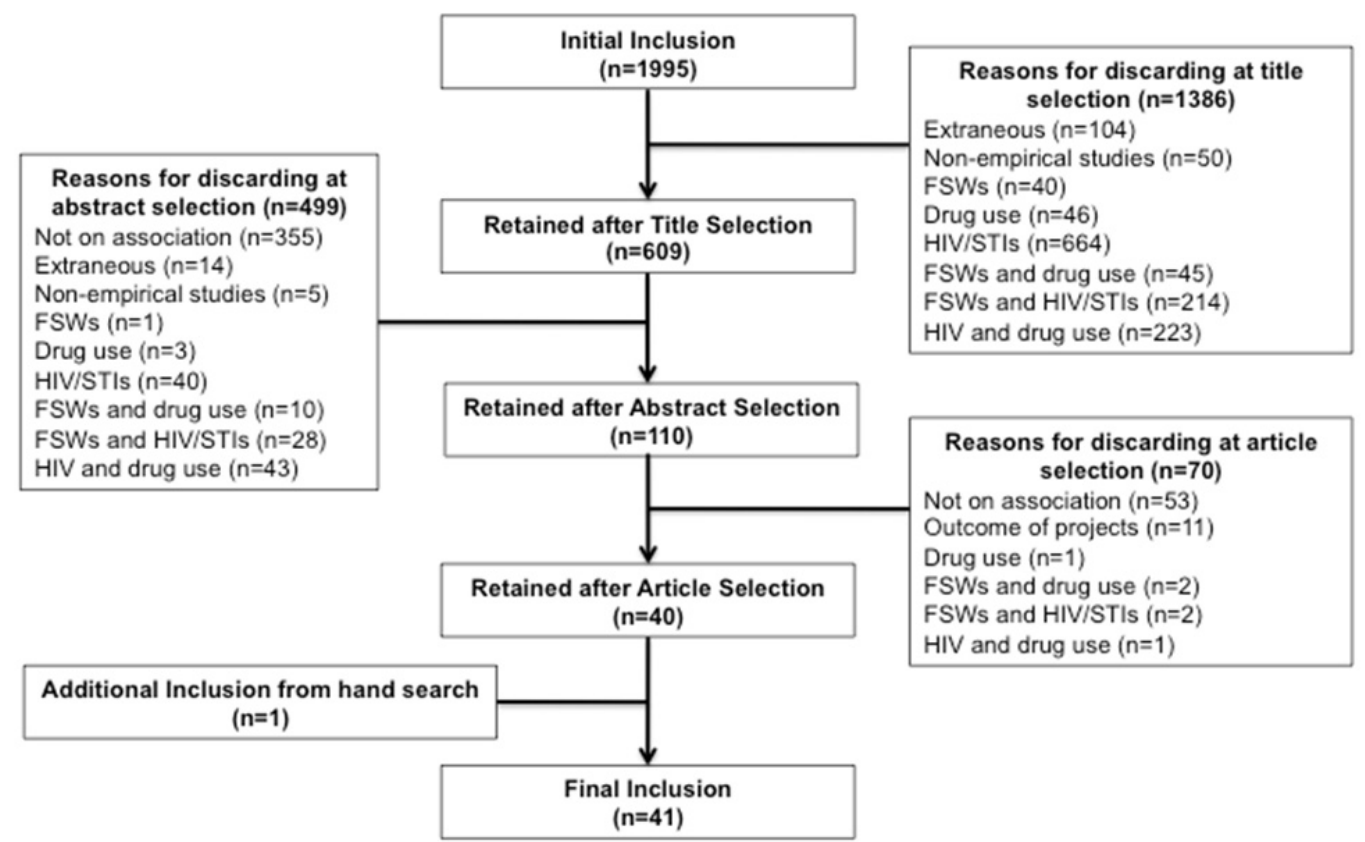

Figure 1. Selection Process

\section{Results}

\subsection{Characteristics of the Reviewed Studies}

\subsubsection{Study Sites and Publication Period}

The key characteristics of the reviewed studies were summarized by authors, publication year, study site, year of data collection, sample size, age of study sample, and study design (Table 1). Our reviewed studies encompassed participants recruited from 16 countries or regions with almost half $(46.3 \%)$ from high-income countries and the other half (53.7\%) from low- and mid-income countries. In particular, data were collected from the United States [ $n=8$ ], Canada [ $n=2]$, the UK [ $n=2]$, The Netherlands [ $n=2]$, Denmark [ $n=1]$, Spain [ $n=3]$, Italy [ $n=1]$, the Russian Federation [ $\mathrm{n}=2$ ], China [ $\mathrm{n}=8$ ], Vietnam [ $\mathrm{n}=2$ ], India [n=1], Bangladesh [ $\mathrm{n}=1]$, Mexico [n=3], Brazil [n=2], South Africa [n=2], and South America [ $\mathrm{n}=1$ ]. The reviewed articles were published between 1989 and 2014, with 13 published prior to 2000, 20 between 2000 and 2009, and eight during or after 2010. The years of data collection ranged between 1985 and 2012.

\subsubsection{Study Design and Sampling}

Most reviewed articles (37 out of 41) adopted quantitative methods and only four used qualitative methods (9.8\%). Most quantitative studies used cross-sectional design (34/37) and only three were cohort studies (Azim et al., 2006; Weber et al., 2002; Vioque et al., 1998). The majority of the studies recruited research participants through STI clinics or other related local community settings (e.g., AIDS outreach offices, HIV-outpatient facilities, national sentinel surveillance sites). Five studies used chain referral sampling (including respondent-driven sampling or RDS) (Medhi et al., 2012; Malta et al., 2008; Gu et al., 2008; Parry et al., 2009; Szwarcwald et al., 1998), and one study used multiple recruitment strategies (Dunne et al., 2014). The sample size (relevant to DU-FSWs) ranged from 10 to 2658 (mean=263.8, median=111). Nineteen studies $(46.3 \%)$ had sample sizes of 100 or less, with eight studies (19.5\%) having sample sizes below 50 .

\subsubsection{Target Population}

As illustrated in Table 1, eight of the 41 (19.5\%) reviewed articles solely focused on drug-using (either injection or non-injection drug using) FSWs. The rest included other high-risk groups in their target populations (e.g., non-sex-trading drug-using women, male IDUs, sex workers' clients). The mean (or at times median) age of the DU-FSWs ranged from 19 to 39.5 (among 19 studies with age information for DU-FSWs). DU-FSWs in the United States were older; mean/median ages of DU-FSWs of United States in all five studies (except three studies from which data on ages were not available) were over 30 years of age. Twenty studies did not report the mean/median age among DU-FSWs, and three studies did not specify the age of their study samples. 
Table 1. Summary of the Reviewed Studies

\begin{tabular}{|c|c|c|c|c|c|c|}
\hline Authors (Year) & $\begin{array}{l}\text { Ref } \\
\#\end{array}$ & Study site & $\begin{array}{l}\text { Year(s) of } \\
\text { data } \\
\text { collection }\end{array}$ & Study sample \& size & Age of sample (mean) & Study design \\
\hline $\begin{array}{l}\text { Dunne et al. } \\
(2014)\end{array}$ & 37 & Baltimore, USA & 2002-2012 & $\begin{array}{l}92 \text { African American DU-FSWs } \\
\text { (sex exchange; } 15 \text { sex traders for } \\
\text { drug only \& } 77 \text { sex traders for } \\
\text { economic resources or both) }\end{array}$ & $\begin{array}{l}37.1 \\
\text { (traders for economic } \\
\text { resources and/or drugs) } \\
\text { vs. } 39.5 \text { (traders for } \\
\text { drugs only) }\end{array}$ & Cross-sectional \\
\hline $\begin{array}{l}\text { Tang et al. } \\
(2014)\end{array}$ & 22 & Guangxi, China & 2010 & $\begin{array}{l}389 \text { DU-FSWs (59 IDU-FSWs and } \\
330 \text { NIDU-FSWs among the total } \\
12,622 \text { FSWs) }\end{array}$ & $\begin{array}{l}\mathrm{n} / \mathrm{a} \\
\text { FSWs })\end{array}$ & Cross-sectional \\
\hline $\begin{array}{l}\text { Hong et al. } \\
(2013)\end{array}$ & 76 & Ningbo, China & 2012 & 63 DU-FSWs (among 863 FSWs) & n/a (21-30 yrs.) & Cross-sectional \\
\hline $\begin{array}{l}\text { Medhi et al. } \\
(2012)\end{array}$ & 2 & Northeast India & 2006 & 107 DU-FSWs (among 426 FSWs) & 26 yrs. & Cross-sectional \\
\hline $\begin{array}{l}\mathrm{Xu} \text { et al. } \\
(2012)\end{array}$ & 38 & Yunnan, China & 2007 & $\begin{array}{l}150 \text { DU-FSWs (among } 705 \text { FSWs } \\
\& 100 \text { clients) }\end{array}$ & 26.7 yrs. & Cross-sectional \\
\hline $\begin{array}{l}\text { Chen et al. } \\
(2012)\end{array}$ & 6 & $\begin{array}{l}\text { Eastern \& southern } \\
\text { China } \\
\text { (eight cities) }\end{array}$ & 2009 & $\begin{array}{l}20 \text { IDU-FSWs (among 7,118 } \\
\text { FSWs) }\end{array}$ & $\begin{array}{l}\mathrm{n} / \mathrm{a} \\
(24 \text { yrs. among all } \\
\text { FSWs)* }\end{array}$ & Cross-sectional \\
\hline $\begin{array}{l}\text { Strathdee et al. } \\
\text { (2011) }\end{array}$ & 56 & $\begin{array}{l}\text { Northern Mexico } \\
\text { (two cities) }\end{array}$ & 2008-2009 & 620 IDUs-FSWs & 33 yrs.* & Cross-sectional \\
\hline $\begin{array}{l}\text { Loza et al. } \\
(2010)\end{array}$ & 23 & $\begin{array}{l}\text { Tijuana \& Ciudad } \\
\text { Juarez, Mexico }\end{array}$ & 2004-2006 & $\begin{array}{l}181 \text { IDU-FSWs (among } 914 \text { FSWs } \\
\text { who had recent unprotected sex) }\end{array}$ & $\begin{array}{l}\mathrm{n} / \mathrm{a} \\
(32 \text { yrs. among all } \\
\text { FSWs })\end{array}$ & Cross-sectional \\
\hline $\begin{array}{l}\text { Wang et al. } \\
(2009)\end{array}$ & 39 & $\begin{array}{l}\text { Yunnan (Kaiyuan } \\
\text { City), China }\end{array}$ & 2006 & $\begin{array}{l}120 \text { DU-FSWs (69 IDU-FSWs \& } \\
51 \text { NIDU-FSWs among } 737 \text { FSWs) }\end{array}$ & $\begin{array}{l}\mathrm{n} / \mathrm{a} \\
(16-52 \text { yrs. })\end{array}$ & Cross-sectional \\
\hline $\begin{array}{l}\text { Parry et al. } \\
(2009)\end{array}$ & 27 & $\begin{array}{l}\text { Cape Town, South } \\
\text { Africa }\end{array}$ & 2005 & $\begin{array}{l}74 \text { DU-FSWs (among } 115 \\
\text { DU-CSWs) }\end{array}$ & $\begin{array}{l}\mathrm{n} / \mathrm{a} \\
(18-55 \mathrm{yrs} .)\end{array}$ & $\begin{array}{l}\text { Qualitative } \\
\text { (rapid } \\
\text { assessment) }\end{array}$ \\
\hline $\begin{array}{l}\mathrm{Xu} \text { et al. } \\
(2008)\end{array}$ & 70 & $\begin{array}{l}\text { Gejiu City, China } \\
\text { (mining regions) }\end{array}$ & 2006 & $\begin{array}{l}10 \text { DU-FSWs (7 IDU-FSWs and } 3 \\
\text { NIDU-FSWs, among } 96 \text { FSWs \& } \\
339 \text { miner clients) }\end{array}$ & $\begin{array}{l}\mathrm{n} / \mathrm{a} \\
(16-39 \text { yrs. among all } \\
\text { FSWs })\end{array}$ & Cross-sectional \\
\hline $\begin{array}{l}\text { Malta et al. } \\
(2008)\end{array}$ & 4 & $\begin{array}{l}\text { Foz do Iguaçu, } \\
\text { Southern Brazil }\end{array}$ & 2003 & 26 NIDU-FSWs (crack users) & $\begin{array}{l}\mathrm{n} / \mathrm{a} \\
(18-40 \text { yrs. })\end{array}$ & Qualitative \\
\hline $\begin{array}{l}\mathrm{Gu} \text { et al. } \\
(2008)\end{array}$ & 20 & $\begin{array}{l}\text { Danzhou } \\
\text { (Sichuan) \& } \\
\text { Hengyang } \\
\text { (Hunan), China }\end{array}$ & 2007 & $\begin{array}{l}281 \text { (non-institutionalized) } \\
\text { IDU-FSWs }\end{array}$ & $\mathrm{n} / \mathrm{a}$ & Cross-sectional \\
\hline $\begin{array}{l}\text { Patterson et al. } \\
(2008)\end{array}$ & 41 & $\begin{array}{l}\text { Tijuana \& Ciudad } \\
\text { Juarez, Mexico }\end{array}$ & 2004-2006 & $\begin{array}{l}424 \text { DU-FSWs (166 IDU-FSWs \& } \\
258 \text { NIDU-FSWs among } 924 \\
\text { FSWs) }\end{array}$ & $\begin{array}{l}\mathrm{n} / \mathrm{a} \\
(32 \text { yrs. among all } \\
\text { FSWs })^{*}\end{array}$ & Cross-sectional \\
\hline
\end{tabular}




\begin{tabular}{|c|c|c|c|c|c|c|}
\hline $\begin{array}{l}\text { Platt et al. } \\
(2007)\end{array}$ & 89 & $\begin{array}{l}\text { Moscow, } \\
\text { Volgograd \& } \\
\text { Barnaul, Russian } \\
\text { Federation }\end{array}$ & 2003 & $\begin{array}{l}104 \text { IDU-FSWs (among } 1473 \\
\text { IDUs) }\end{array}$ & $21.5 \mathrm{yrs}$ & Cross-sectional \\
\hline $\begin{array}{l}\text { Bautista et al. } \\
\text { (2006) }\end{array}$ & 90 & $\begin{array}{l}\text { South America } \\
\text { (nine countries) }\end{array}$ & $1999-2002$ & $\begin{array}{l}16 \text { DU-FSWs (10 illegal } \\
\text { drug-using FSWs \& } 6 \text { marijuana } \\
\text { using FSWs among 13,600 FSWs) }\end{array}$ & $\begin{array}{l}\mathrm{n} / \mathrm{a} \\
(28.3 \text { yrs. among all } \\
\text { FSWs })\end{array}$ & Cross-sectional \\
\hline$\underset{(2006)}{\text { Azim }}$ et al. & 33 & $\begin{array}{l}\text { Dhaka, } \\
\text { Bangladesh (three } \\
\text { cities) }\end{array}$ & 2004-2005 & $\begin{array}{l}82 \text { IDU-FSWs (among } 130 \text { female } \\
\text { IDUs) }\end{array}$ & 27 yrs.* & $\begin{array}{l}\text { Cohort study } \\
\text { (baseline data) }\end{array}$ \\
\hline $\begin{array}{l}\text { Inciardi et al. } \\
(2006)\end{array}$ & 5 & $\begin{array}{l}\text { Miami, Florida, } \\
\text { USA }\end{array}$ & $2001-2003$ & 586 DU-FSWs (street based) & 38 yrs.* & Cross-sectional \\
\hline $\begin{array}{l}\text { Chen et al. } \\
(2005)\end{array}$ & 44 & $\begin{array}{l}\text { Kunming, Yunnan, } \\
\text { China }\end{array}$ & $1999-2000$ & 292 DU-FSWs (among 505 FSWs) & $\begin{array}{l}\mathrm{n} / \mathrm{a} \\
(24.7 \text { yrs. among all } \\
\text { FSWs })\end{array}$ & Cross-sectional \\
\hline
\end{tabular}

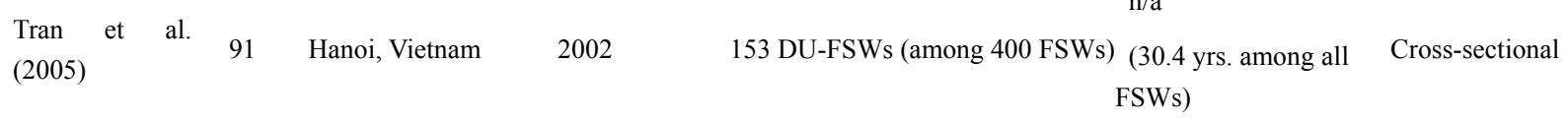

\begin{tabular}{|c|c|c|c|c|c|c|}
\hline $\begin{array}{l}\text { Platt et al. } \\
(2005)\end{array}$ & 17 & $\begin{array}{l}\text { Togliatti City, } \\
\text { Russian Federation }\end{array}$ & 2001 & 66 IDU-FSWs (among 423 IDUs) & 23.8 yrs. & Cross-sectional \\
\hline $\begin{array}{l}\text { Belza } \\
(2004)\end{array}$ & 92 & $\begin{array}{l}\text { Spain } \\
\text { (six cities) }\end{array}$ & 2000 & $\begin{array}{l}19 \text { IDU-FSWs (among } 3149 \\
\text { FSWs) }\end{array}$ & $\begin{array}{l}\mathrm{n} / \mathrm{a} \\
(29.2 \text { yrs. among all } \\
\text { FSWs })\end{array}$ & Cross-sectional \\
\hline
\end{tabular}

\begin{tabular}{|c|c|c|c|c|c|c|}
\hline $\begin{array}{l}\text { Nguyen et al. } \\
(2004)\end{array}$ & 45 & $\begin{array}{l}\text { Ho Chi Minh City, } \\
\text { Vietnam }\end{array}$ & 2000 & $\begin{array}{l}51 \text { IDU-FSWs (among } 398 \\
\text { street-based FSWs) }\end{array}$ & $25.4 \mathrm{yrs}$. & Cross-sectional \\
\hline $\begin{array}{l}\text { Spittal et al. } \\
(2003)\end{array}$ & 42 & $\begin{array}{l}\text { Vancouver \& } \\
\text { Montreal, Canada }\end{array}$ & $1999-2000$ & $\begin{array}{l}193 \text { IDU-FSWs (among } 591 \\
\text { street-based female IDUs) }\end{array}$ & 31 yrs.* & Cross-sectional \\
\hline $\begin{array}{l}\text { Weber et al. } \\
(2002)\end{array}$ & 34 & Montreal, Canada & $1995-2000$ & $\begin{array}{l}88 \text { DU-FSWs (among } 265 \text { female } \\
\text { street youths) }\end{array}$ & 19 yrs. & $\begin{array}{l}\text { Prospective } \\
\text { cohort }\end{array}$ \\
\hline $\begin{array}{l}\text { Leggett et al. } \\
(2001)\end{array}$ & 14 & $\begin{array}{l}\text { Johannesburg, } \\
\text { Cape Town, \& } \\
\text { Durban, South } \\
\text { Africa }\end{array}$ & $\mathrm{n} / \mathrm{a}$ & $\begin{array}{l}\text { Hard-drug-using FSWs (among } \\
349 \text { street FSWs) }\end{array}$ & $\begin{array}{l}\text { n/a } \\
\text { (Johannesburg: } 24 \\
\text { yrs. } \\
\text { Cape Town: } 27 \text { yrs. } \\
\text { Durban: } 27 \text { yrs.; } \\
\text { among all FSWs) }\end{array}$ & Cross-sectional \\
\hline $\begin{array}{l}\text { Kwiatkowski \& } \\
\text { Booth (2000) }\end{array}$ & 96 & 23 cities, USA & 1992-1996 & $\begin{array}{l}\text { 2,042 DU-FSWs (sex exchangers } \\
\text { for money or drugs) }\end{array}$ & 35 yrs. & Cross-sectional \\
\hline $\begin{array}{l}\text { Logan } \quad \& \\
\text { Leukefeld } \\
(2000)\end{array}$ & 93 & 20 sites, USA & 1992-1997 & $\begin{array}{l}2658 \text { crack-using FSWs (among } \\
4667 \text { crack-using women) }\end{array}$ & 37 yrs. & Cross-sectional \\
\hline $\begin{array}{l}\text { Estébanez et al. } \\
\text { (1998) }\end{array}$ & 94 & $\begin{array}{l}22 \text { sites ( } 29 \\
\text { provinces), Spain }\end{array}$ & 1989-1991 & $\begin{array}{l}269 \text { IDU-FSWs (among } 1633 \\
\text { FSWs) }\end{array}$ & $\begin{array}{l}\mathrm{n} / \mathrm{a} \\
(29.8 \text { yrs. among all } \\
\text { FSWs })\end{array}$ & Cross-sectional \\
\hline $\begin{array}{l}\text { Vioque et al. } \\
\text { (1998) }\end{array}$ & 35 & Alicante, Spain & 1986-1996 & 50 IDU-FSWs (among 657 FSWs) & $\begin{array}{l}\mathrm{n} / \mathrm{a} \\
(27 \text { yrs. among all } \\
\text { FSWs })\end{array}$ & $\begin{array}{l}\text { Prospective } \\
\text { cohort }\end{array}$ \\
\hline
\end{tabular}




\begin{tabular}{|c|c|c|c|c|c|c|}
\hline $\begin{array}{l}\text { Szwarcwald et } \\
\text { al. (1998) }\end{array}$ & 36 & Santos, Brazil & 1996 & $\begin{array}{l}22 \text { IDU-FSWs (among } 697 \\
\text { chain-referred FSWs) }\end{array}$ & 27.4 yrs. & Cross-sectional \\
\hline $\begin{array}{l}\text { Logan et al. } \\
(1998)\end{array}$ & 43 & $\begin{array}{l}\text { Lexington \& } \\
\text { Louisville, } \\
\text { Kentucky, USA }\end{array}$ & 1993-1997 & $\begin{array}{l}162 \text { crack-using FSWs (sex } \\
\text { exchangers among } 292 \text { female } \\
\text { crack users) }\end{array}$ & 35 yrs. & Cross-sectional \\
\hline $\begin{array}{l}\text { Jones et al. } \\
(1998)\end{array}$ & 47 & $\begin{array}{l}\text { Miami, New York } \\
\text { City, San } \\
\text { Fransicso, USA }\end{array}$ & 1991-1992 & $\begin{array}{l}337 \text { crack-using FSWs (among } \\
419 \text { crack-smoking CSWs) }\end{array}$ & $\begin{array}{l}\mathrm{n} / \mathrm{a} \\
(18-29 \mathrm{yrs} .)\end{array}$ & Cross-sectional \\
\hline $\begin{array}{r}\text { Inciardi } \\
(1995)\end{array}$ & 46 & $\begin{array}{l}\text { Miami, Florida, } \\
\text { USA }\end{array}$ & $1989-1993$ & $\begin{array}{l}35 \text { crack-using FSWs (among } 52 \\
\text { crack-users who exchanged sex for } \\
\text { crack) }\end{array}$ & $\begin{array}{l}\mathrm{n} / \mathrm{a} \\
(19-40 \mathrm{yrs})\end{array}$ & Qualitative \\
\hline $\begin{array}{l}\text { Alary et al. } \\
(1994)\end{array}$ & 48 & $\begin{array}{l}\text { Copenhagen, } \\
\text { Denmark }\end{array}$ & $1990-1991$ & $\begin{array}{l}36 \text { IDU-FSWs (including } 237 \\
\text { FSWs) }\end{array}$ & $\begin{array}{l}\mathrm{n} / \mathrm{a} \\
(30.9 \text { yrs. among all } \\
\text { FSWs })\end{array}$ & Cross-sectional \\
\hline $\begin{array}{l}\text { Astemborski } \\
\text { and Solomon } \\
(1994)\end{array}$ & 52 & Baltimore, USA & $1988-1989$ & $\begin{array}{l}211 \text { IDU-FSWs (women who } \\
\text { traded sex for drugs or money } \\
\text { among } 538 \text { female IDUs) }\end{array}$ & $\mathrm{n} / \mathrm{a}$ & Cross-sectional \\
\hline $\begin{array}{l}\text { Taylor et al. } \\
(1993)\end{array}$ & 50 & Glasgow, UK & $1990-1991$ & 51 IDU-FSWs & 25.5 yrs. & Cross-sectional \\
\hline $\begin{array}{l}\text { McKeganey et } \\
\text { al. (1992) }\end{array}$ & 87 & Glasgow, UK & 1991 & $\begin{array}{l}147 \text { IDU-FSWs (among } 206 \\
\text { street-working FSWs) }\end{array}$ & $\mathrm{n} / \mathrm{a}$ & $\begin{array}{l}\text { Qualitative } \\
+ \text { HIV tests }\end{array}$ \\
\hline $\begin{array}{l}\text { van den Hoek } \\
\text { et al. (1990) }\end{array}$ & 95 & $\begin{array}{l}\text { Amsterdam, the } \\
\text { Netherlands }\end{array}$ & 1985 & 95 IDU-FSWs (among 243 IVDS) & $\begin{array}{l}\mathrm{n} / \mathrm{a} \\
(28.6 \text { yrs. among } \\
\text { female IDUs) }\end{array}$ & Cross-sectional \\
\hline $\begin{array}{l}\text { van den Hoek } \\
\text { et al. (1989) }\end{array}$ & 49 & $\begin{array}{l}\text { Amsterdam, the } \\
\text { Netherlands }\end{array}$ & $1985-1987$ & $\begin{array}{l}117 \text { DU-FSWs (96 IDU-FSWs \& } \\
21 \text { NIDU-FSWs) }\end{array}$ & 27 yrs. & Cross-sectional \\
\hline $\begin{array}{l}\text { Tirelli et al. } \\
(1989)\end{array}$ & 62 & $\begin{array}{l}\text { Rome, Milan, } \\
\text { Naples, } \\
\text { Pordenone, Italy }\end{array}$ & $\mathrm{n} / \mathrm{a}$ & $\begin{array}{l}114 \text { IDU-FSWs (among } 304 \\
\text { FSWs) }\end{array}$ & 25.6 yrs.* & Cross-sectional \\
\hline
\end{tabular}

* Median age.

\subsection{HIV Risk}

\subsubsection{HIV Prevalence}

The prevalence of HIV infection among DU-FSWs varied substantially across study samples within various geographical regions. The reviewed studies examined HIV rates (e.g., seroprevalence, seropositivity, seroconversion) among IDU-FSWs $(0-56 \%$, mean $=24.2 \%$, median $=16.3 \%$, among 19 studies $)$, NIDU-FSWs $(0-60 \%$, mean $=23.8 \%$, median $=20.3 \%$, among 6 studies $)$, and FSWs who used both injection drugs and non-injection drugs $(4.8-43.4 \%$, mean $=21.9 \%$, median $=23.7 \%$, among ten studies).

Our reviewed articles yielded a general pattern regarding the level of association between drug use and HIV prevalence among FSWs. The rates of HIV infection were highest among IDU-FSWs, the next highest among NIDU-FSWs, and the lowest among non-DU-FSWs (Table 2; HIV rates were calculated to represent the prevalence among DU-FSWs when the study population was larger than DU-FSWs and the relevant data was available). Six studies show that IDU-FSWs had much higher HIV prevalence than NIDU-FSWs (Medhi et al., 2012; Tang et al., 2014; Szwarcwald et al., 1998; Xu et al., 2012; Wang et al., 2009; Tran, et al., 2005). More specifically, in Kaiyuan City, China, IDU-FSWs had the highest rate of HIV infection (49.3\%), followed by DU-FSWs (19.6\%), whereas the HIV prevalence among FSWs overall was 10.3\% (Wang et al., 2009). In Santos, Brazil, IDU-FSWs had a $45 \%$ HIV infection rate compared to crack users, who had $38 \%$ HIV prevalence, and marijuana/cocaine users, who had a 5\% HIV infection rate (Szwarcwald et al., 1998). In northeast India and southwest China (Yunnan province), HIV prevalence was $25.2 \%$ and $43.4 \%$ among DU-FSWs and $9.5 \%$ and $4.9 \%$ 
among non-DU-FSWs, respectively (Medhi et al., 2012; Xu et al., 2012). The only exception to this general pattern was in Mexico, where NIDU-FSWs had a $60.0 \%$ HIV rate whereas IDU-FSWs had a $41.8 \%$ rate (Patterson et al., 2008).

\subsubsection{STI Prevalence}

Reviewed studies also examined various other STIs among IDU-FSWs. For instance, among IDU-FSWs, syphilis infection rates ranged from $17 \%$ to $58 \%$ (mean $=32.9 \%$, median $=25.0 \%$, among 5 studies). Other measures of syphilis such as syphilis tiers $\geq 1.8(36 \%)$, lifetime syphilis $(60 \%)$, and active syphilis $(10.7 \%)$ were also reported. Two studies reported gonorrhea infection (6-65\%, mean=35.5\%). HCV (18.7\%), HBV (41.8\%), Chlamydia (24\%), Trichomoniasis (58\%), genital herpes (24\%), bacterial vaginosis (42\%), treponema pallidum (16.3\%), HTLV-I $(0 \%)$, and HTLV-II (53\%) were also examined and reported. Among NIDU-FSWs, syphilis $(4.5 \%)$ and HCV $(1.5 \%)$ were examined.

Reviewed articles that included both IDU-FSWs and NIDU-FSWs examined syphilis $(12-40 \%$, mean $=23.2 \%$, median $=22 \%$, among 7 studies), Chlamydia $(10.1-30 \%$, mean $=18.8 \%$, median $=17.5 \%$, among 4 studies), gonorrhea $(6.1-44 \%$, mean $=30 \%$, median $=40 \%$, among 3 studies), HCV $(22.2-29.7 \%$, mean $=26 \%$, among 2 studies), HBV (53.4-67\%, mean=60.2\%, among 2 studies), genital warts $(6-8 \%$, mean $=7 \%$, among 2 studies), HSV-2 (73-86.7\%, mean $=79.9 \%$, among 2 studies), Trichomoniasis (9.5\%), and any STI (59\%). In addition, a few studies used other variables such as the infection histories of STIs (3-8.5\%, mean $=6.3 \%$, among 2 studies).

\subsubsection{Drug-Related HIV Risk}

The majority of the reviewed studies (82.9\%) included DU-FSWs as part of their primary subjects. As to drug use patterns among DU-FSWs, nearly half of the studies (43.9\%) solely focused on IDU-FSWs, nearly half (41.5\%) studied both IDU-FSWs and NIDU-FSWs, only a few (4.9\%) focused on NIDU-FSWS, and some (9.8\%) did not distinguish between IDU-FSWs and NIDU-FSWs at all.

Injection drug use and non-injection drug use were often not exclusive, and poly-drug use was common among FSWs. For instance, Spittal et al., (Spittal et al., 2003) reported that IDU-FSWs in Vancouver and Montreal, Canada, used crack, heroin, and speedball along with injection drugs. Logan et al., (Logan, Leukefeld, Farabee, 1998) mentioned that $51 \%$ of the crack-using FSWs in Lexington and Louisville, Kentucky US, were also IDUs concurrently. Non-injection drug use among FSWs included the use of opioids, cocaine, heroin, crack, methamphetamine, ketamine, marijuana, ecstasy, cannabis, and speedball.

As to particular drug-related risk practices for HIV transmission, the reviewed articles largely reported two kinds of behaviors: IDU-related risk behavior (28/41) and NIDU-related risk behavior (6/41). The three most frequent risk factors were: IDU (7.6-100\%, mean $=48.7 \%$, median $=44.2 \%, 16$ studies), needle/equipment sharing (3.2-74.4\%, mean $=43.3 \%$, median $=48.4 \%, 8$ studies), and NIDU $(82.6-100 \%$, mean $=92.6 \%$, median $=90.6 \%, 5$ studies). The articles also showed various associations between HIV/STI status and FSWs' demographic characteristics (e.g., age at time of study, age at first involvement in illicit activities, STI history, type of work venues) that were unique to each setting.

Depending on various sampling frames, the proportion of IDU among DU-FSWs in reviewed studies ranged from $3 \%$ to $100 \%$ (mean $=69.3 \%$, median $=82.6 \%$, 34 studies), and the proportion of NIDU ranged from $17 \%$ to $90.6 \%$ (mean $=58.7 \%$, median $=69.2 \%, 10$ studies). The DU-FSWs used various kinds of drugs including crack $(72-100 \%$, mean $=91.1-100 \%$, median $=100 \%$, 7 studies), heroin $(35-57 \%$, mean $=49.3 \%$, median $=56 \%, 3$ studies $)$, marijuana $(37.5-100 \%$, mean $=77.5 \%$, median $=95 \%, 3$ studies $)$, cocaine $(38-93 \%$, mean $=65.5 \%, 2$ studies $)$, opioids $(29.3 \%)$, methamphetamine (92\%), ketamine (19\%), speedball (33\%), acid/PCP (94\%), tranquilizers $(68 \%)$, and solvents $(51 \%)$. Because the line between IDU and NIDU was often not made clear, calculations in this review were only based on the available data from the reviewed studies.

Injection drug use among FSWs was often the greatest risk factor for contracting HIV. For example, a study in Kunming, China, showed that all HIV-infected FSWs (10\% of the studied population) were IDUs (Chen et al., 2005). In Ho Chi Minh City, Vietnam, injection drug use was independently associated with HIV seropositivity $(\mathrm{OR}=101.30 ; 95 \% \mathrm{CI}, 33.55-3-5.89)$ (Nguyen et al., 2004). In Guangxi province, China, the HIV rate among IDU-FSWs was $8.5 \%$ whereas that of other FSWs was $1.0 \%$ or less in the 2010 National Sentinel Surveillance sample (Tang et al., 2014).

However, studies also showed that non-injection drug use was a risk factor facilitating HIV transmission among FSWs. Drug use (often "binge use" or use at an overdose level) led to impaired host immunity, which potentiated the transmission of HIV as well as STIs (such as genital ulceration), by increasing both susceptibility and infectivity (Inciardi 1995). Crack use, for example, can cause open sores, blisters, and cuts on lips and inside 
mouths, and the wounds may facilitate the oral transmission of HIV during pipe sharing (Malta et al., 2008) or oral sex (Inciardi 1995).

Three reviewed studies have suggested that non-injection drug use (compared with injection drugs) may equally be a risk factor for HIV infection (Medhi et al., 2012; Inciardi 1995). Particularly, crack smokers may be at equal or even greater risk for HIV/STIs and play a unique role in transmitting STIs (Inciardi 1995). Interestingly, these studies showed that, while it was true that IDU-FSWs usually had a much higher prevalence of HIV, NIDU-FSWs tended to have a higher prevalence of some other STIs. For example, crack-smoking FSWs were more likely to have syphilis (42\%) than IDU-FSWs (24\%) in the U.S. (Jones et al., 1998).

\subsubsection{Sex-Related HIV Risk}

As shown in Table 2, the reviewed studies largely investigated five sex-related risk factors including: unprotected sex (27/41), infections of other STIs (13/41), number of sex partners (6/41), other risky sex practices (e.g., sex under drug use, serial sex, anal sex) (5/41), and adverse social conditions and environments (e.g., work venues, violence, homelessness, arrest, and imprisonment) (11/41).

Table 2. Summary of HIV-related risk in the reviewed studies

\begin{tabular}{|c|c|c|c|c|c|c|}
\hline $\begin{array}{l}\text { Authors } \\
\text { (Year of } \\
\text { Study) }\end{array}$ & $\begin{array}{l}\mathrm{Re} \\
\mathrm{f \#}\end{array}$ & Drug use patterns & HIV prevalence & Other STI rates & $\begin{array}{l}\text { Drug-related risk } \\
\text { factors }(\%)\end{array}$ & Sex-related risk factors $(\%)$ \\
\hline $\begin{array}{l}\text { Dunne et al. } \\
\text { (2014) }\end{array}$ & 37 & $\begin{array}{l}\text { IDU-FSWs: } 47.8 \% \\
\text { Opioid-use: } 29.3 \% \\
\text { Cocaine-use: } 38.0 \% \\
\text { Crack-use: } 84.8 \%\end{array}$ & $\begin{array}{l}20 \% \text { (traded } \text { sex } \\
\text { for drugs only) } \\
5.2 \% \text { (traded } \text { sex } \\
\text { for } \quad \text { economic } \\
\begin{array}{l}\text { resources } \\
\text { drugs) }\end{array}\end{array}$ & & $\begin{array}{l}\text { IDU, sharing } \\
\text { needles, } \\
\text { opioids/cocaine/crack } \\
\text { use }(\mathrm{n} / \mathrm{a})\end{array}$ & $\begin{array}{l}\text { Number of sex partners, inconsistent } \\
\text { condom use, lifetime condom use rate } \\
\text { (n/a) }\end{array}$ \\
\hline $\begin{array}{l}\text { Tang et al. } \\
(2014)\end{array}$ & 22 & $\begin{array}{l}\text { IDU-FSWs: } 15.2 \% \\
\text { NIDU-FSWs: } 84.8 \%\end{array}$ & $\begin{array}{l}\text { IDU-FSWs: } 8.5 \% \\
\text { NIDU-FSWs: } \\
0.0 \% \\
\text { (non-DU-FSWs: } \\
1.0 \% \text { ) }\end{array}$ & $\begin{array}{l}\text { Syphilis: } 20.3 \% \\
\text { (IDU-FSWs), } \\
4.5 \% \\
\text { (NIDU-FSWs) } \\
\text { HCV: } \quad 44.1 \% \\
\text { (IDU-FSWs), } \\
1.5 \% \\
\text { (NIDU-FSWs) }\end{array}$ & $\mathrm{n} / \mathrm{a}$ & $\begin{array}{l}\text { IDU-FSWs: } \\
\text { No condom use during last sex act: } \\
25.9 \% \\
\text { Inconsistent condom use (past mo.): } \\
64.4 \% \\
\text { STD history (self-report): } 8.5 \% \\
\text { HIV testing: } 61.0 \% \\
\text { NIDU-FSWs: } \\
\text { No condom use during last sex act: } \\
15.7 \% \\
\text { Inconsistent condom use (past mo.): } \\
31.1 \% \\
\text { Self reported history of STDs: } 7.3 \% \\
\text { HIV testing: } 44.2 \%\end{array}$ \\
\hline
\end{tabular}

\section{IDU-FSWs: $22.2 \%$}

\begin{tabular}{|c|c|c|c|c|c|c|}
\hline \multirow{5}{*}{$\begin{array}{l}\text { Hong et al. } \\
\text { (2013) }\end{array}$} & & \multicolumn{2}{|l|}{ Methamphetamine-using } & \multicolumn{2}{|r|}{ Needle sharing } & \multirow[b]{2}{*}{ No condom use (last sex act): $88.9 \%$} \\
\hline & & FSWs: $92.1 \%$ & & \multicolumn{2}{|c|}{ Syphilis: $25.4 \%(3.2 \%)$} & \\
\hline & 76 & Heroin-using FSWs: & $4.8 \%$ & Hepatitis & C: IDU $(22.2 \%)$ & HIV/syphilis/hepatitis C infections \\
\hline & & & & $22.2 \%$ & Methamphetamine/he & \\
\hline & & $\begin{array}{l}\text { Ketamine-using FSWs: } \\
19 \%\end{array}$ & & & & \\
\hline
\end{tabular}




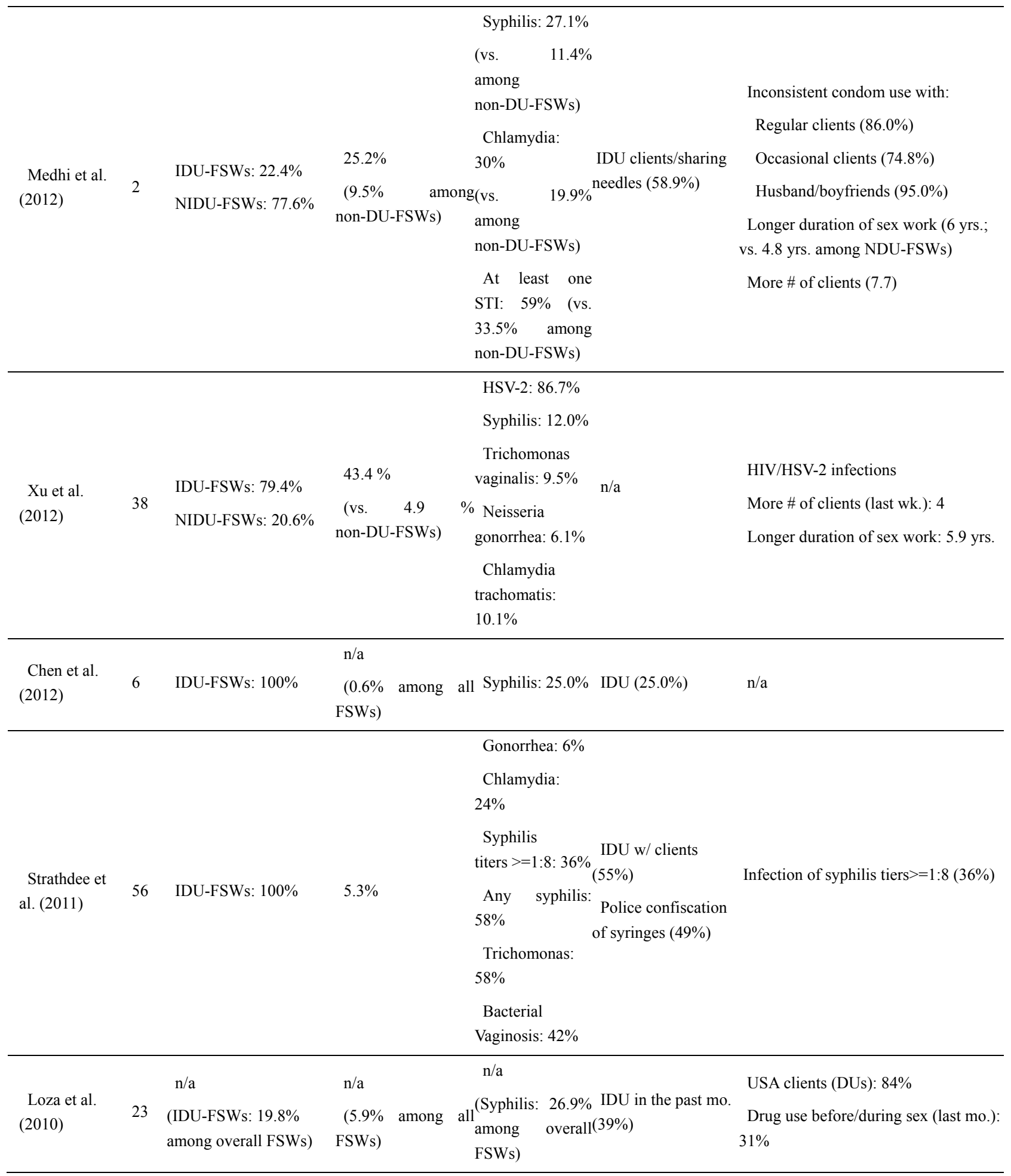




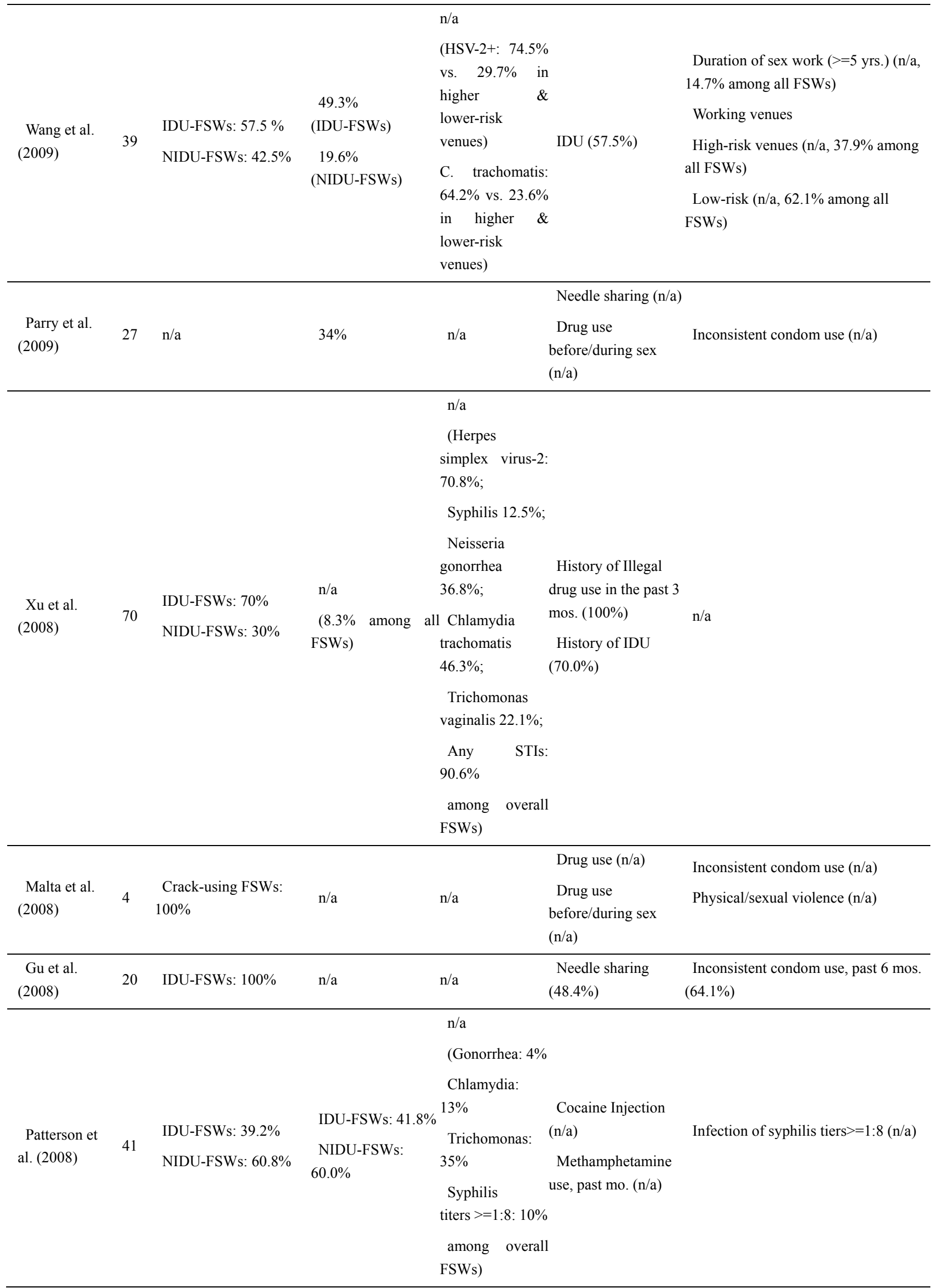




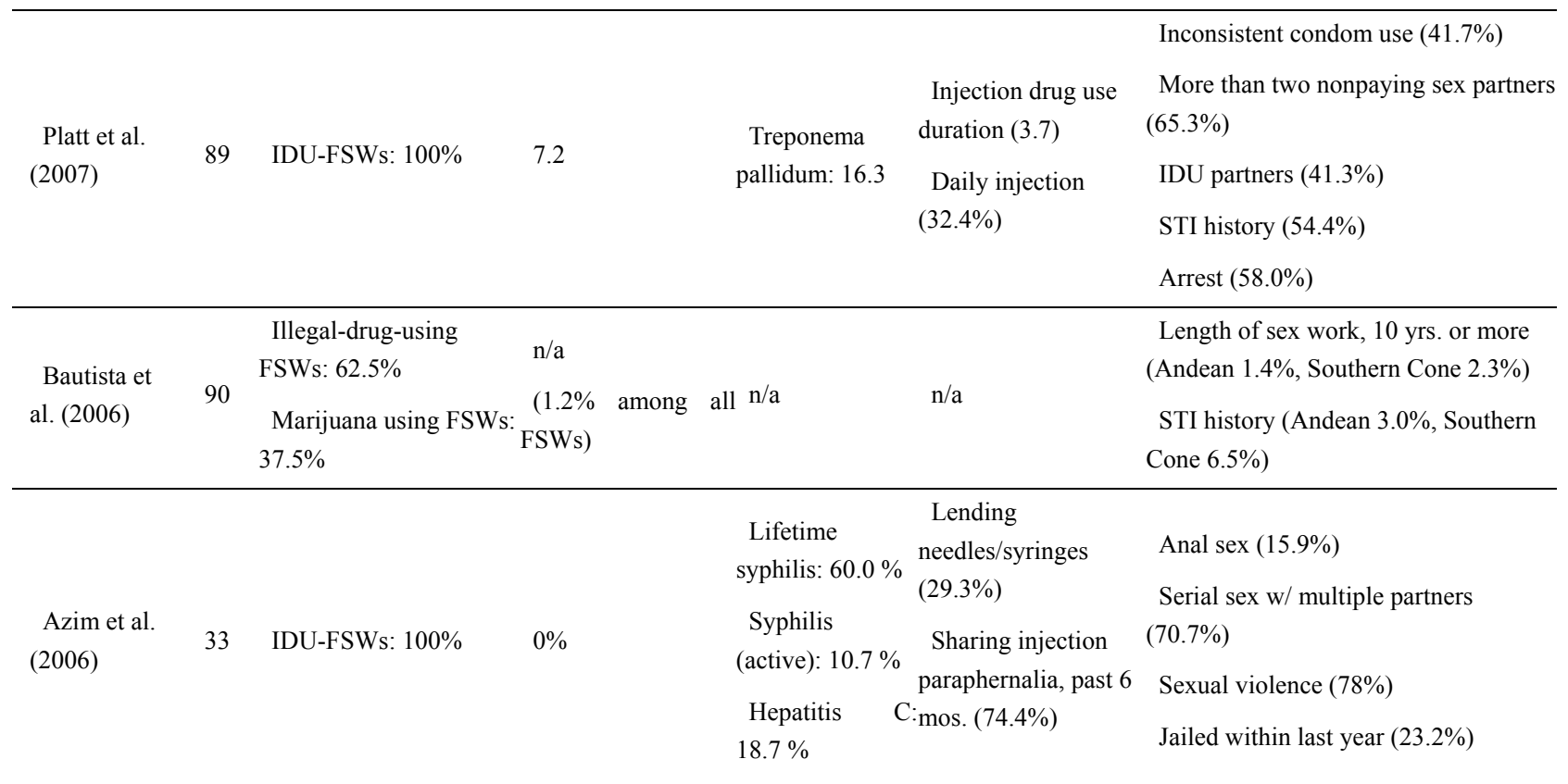

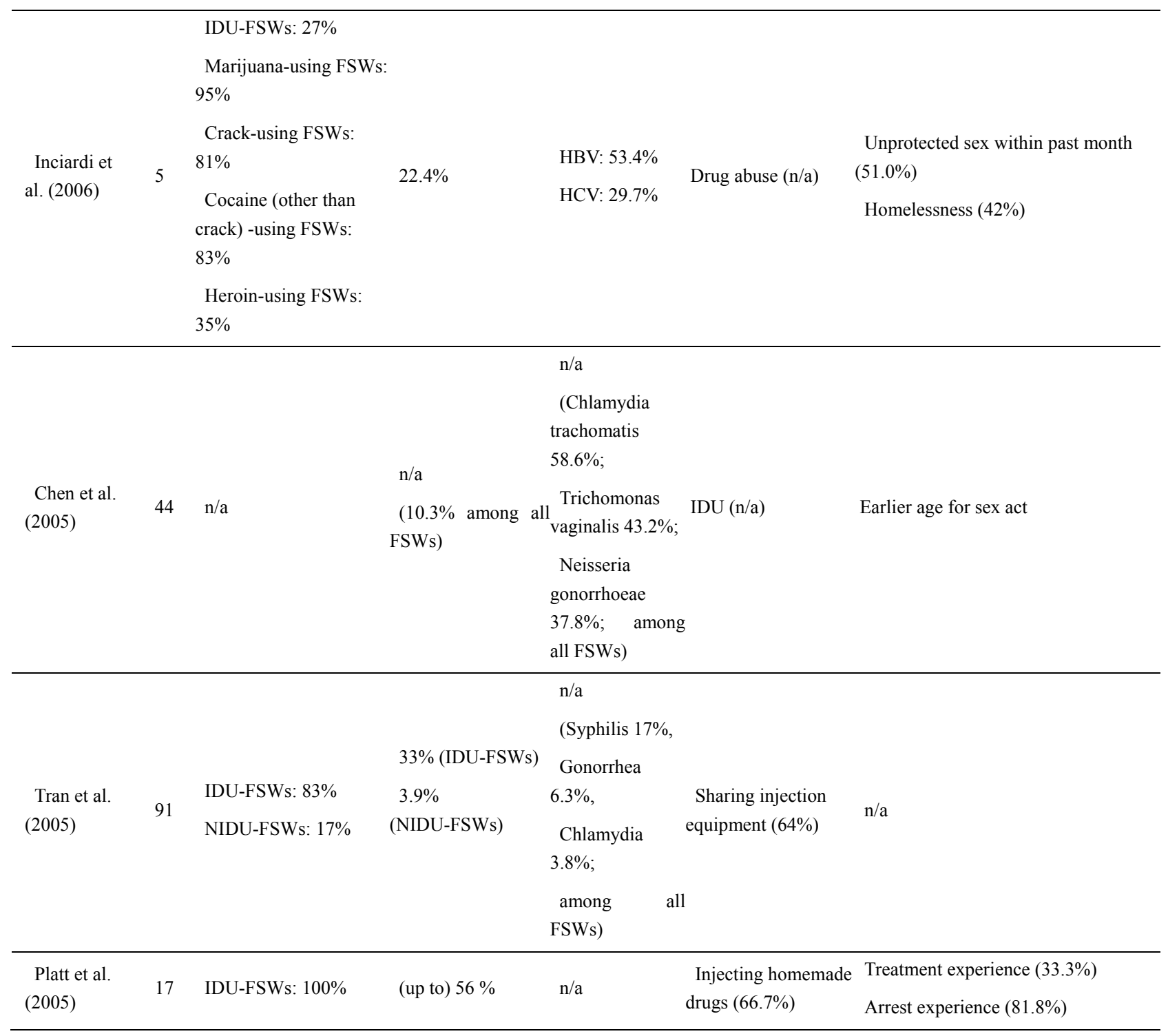




\begin{tabular}{|c|c|c|c|c|c|}
\hline $\begin{array}{r}\text { Belza } \\
(2004)\end{array}$ & 92 & IDU-FSWs: $100 \%$ & 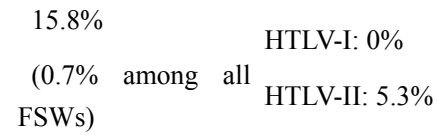 & $\mathrm{n} / \mathrm{a}$ & $\mathrm{n} / \mathrm{a}$ \\
\hline $\begin{array}{l}\text { Nguyen et } \\
\text { al. (2004) }\end{array}$ & 45 & IDU-FSWs: $100 \%$ & $\begin{array}{l}16.3 \% \\
\text { (seroprevalence) }\end{array}$ & IDU & $\mathrm{n} / \mathrm{a}$ \\
\hline $\begin{array}{l}\text { Spittal et al. } \\
(2003)\end{array}$ & 42 & $\begin{array}{l}\text { IDU-FSWs } \\
\text { (intravenous): } 100 \% \\
\text { (crack, heroin, } \\
\text { speedball use as well) }\end{array}$ & $\begin{array}{l}29 \% \\
(29.2 \% \text { among } \mathrm{n} / \mathrm{a} \\
\text { female IDUs })\end{array}$ & $\begin{array}{l}\text { Borrowing used } \\
\text { syringes (35.2\%) } \\
\text { Crack cocaine use } \\
(42.5 \%) \\
\text { Daily heroin use } \\
(37.8 \%)\end{array}$ & $\mathrm{n} / \mathrm{a}$ \\
\hline
\end{tabular}

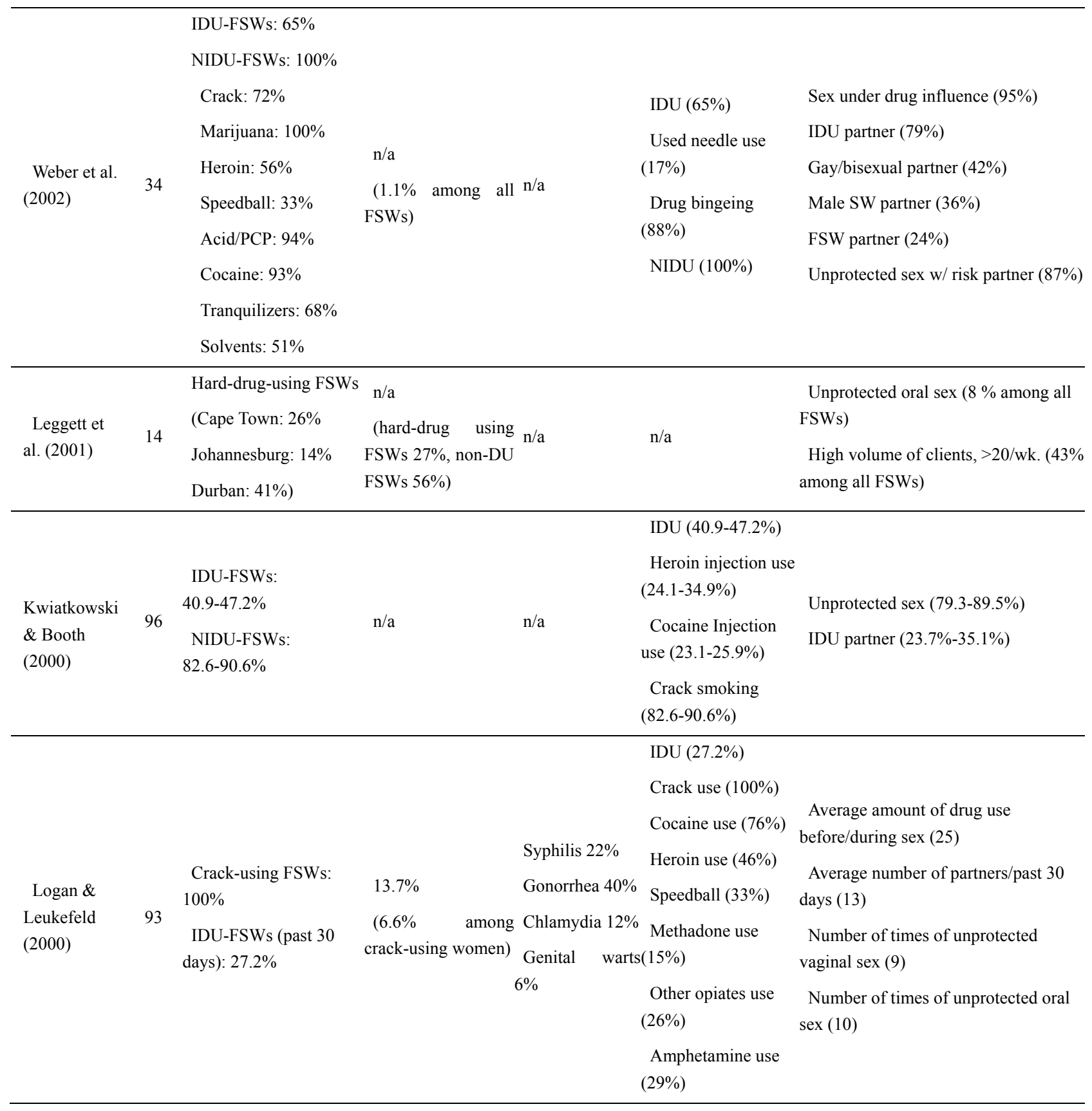




\begin{tabular}{|c|c|c|c|c|c|c|}
\hline $\begin{array}{l}\text { Estébanez } \\
\text { et al. (1998) }\end{array}$ & 94 & IDU-FSWs: $100 \%$ & $\begin{array}{l}51.7 \% \\
(3.7 \% \text { among non- } \\
\text { IDU-FSWs })\end{array}$ & $\begin{array}{l}\text { Hepatitis } \quad \text { B } \\
-41.8 \%\end{array}$ & $\mathrm{n} / \mathrm{a}$ & $\begin{array}{l}\text { IDU partner (n/a, 22.4\% among all } \\
\text { FSWs) } \\
\text { Hepatitis B (n/a, } 41.8 \% \text { among all } \\
\text { FSWs) } \\
\text { Previous imprisonment (n/a, } 13.8 \% \\
\text { among all FSWs) }\end{array}$ \\
\hline $\begin{array}{l}\text { Vioque et } \\
\text { al. (1998) }\end{array}$ & 35 & IDU-FSWs: $100 \%$ & $16 \%$ & $\mathrm{n} / \mathrm{a}$ & IDU $(7.6 \%)$ & IDU partner $(6.4 \%)$ \\
\hline $\begin{array}{l}\text { Szwarcwald } \\
\text { et al. (1998) }\end{array}$ & 36 & $\begin{array}{l}\mathrm{n} / \mathrm{a} \\
\text { (IDU-FSWs: } 3 \% \\
\text { Crack-using FSWs: } \\
6.6 \% \\
\text { Cocaine-using FSWs: } \\
13 \% \\
\text { Marijuana using FSWs: } \\
16 \% \\
\text { among overall FSWs) }\end{array}$ & $\begin{array}{l}45 \% \text { (IDUs) } \\
38 \% \text { (crack users) }\end{array}$ & $\mathrm{n} / \mathrm{a}$ & $\begin{array}{l}\text { Crack use, previous } \\
\text { mo. (n/a) } \\
\text { IDU }(\%)\end{array}$ & Unprotected sex $(\mathrm{n} / \mathrm{a})$ \\
\hline $\begin{array}{l}\text { Logan et al. } \\
(1998)\end{array}$ & 43 & $\begin{array}{l}\text { Crack-using FSWs (sex } \\
\text { exchange): } 100 \% \\
\text { (51\% were also } \\
\text { IDU-FSWs) }\end{array}$ & $\mathrm{n} / \mathrm{a}$ & $\begin{array}{l}\text { Gonorrhea: } \\
44 \% \\
\text { Syphilis: } 16 \% \\
\text { Genital warts: } \\
8 \% \\
\text { Chlamydia: } \\
23 \%\end{array}$ & $\mathrm{n} / \mathrm{a}$ & $\mathrm{n} / \mathrm{a}$ \\
\hline $\begin{array}{l}\text { Jones et al. } \\
\text { (1998) }\end{array}$ & 47 & $\begin{array}{l}\text { IDU-FSWs: } 10.1 \% \\
\text { NIDU-FSWs: } 89.9 \%\end{array}$ & $25 \%$ & $\begin{array}{l}\text { Syphilis: } 40 \% \\
\text { HSV-2: } 73 \%\end{array}$ & $\mathrm{n} / \mathrm{a}$ & $\begin{array}{l}\text { Unprotected sex w/ clients, past } 30 \\
\text { days }(\mathrm{n} / \mathrm{a}) \\
\text { IDU sex partner }(74 \%) \\
\text { HIV-infected partner }(18 \%) \\
\text { Sexual violence }(24 \%)\end{array}$ \\
\hline $\begin{array}{l}\text { Inciardi } \\
(1995)\end{array}$ & 46 & $\begin{array}{l}\text { Crack-using FSWs (sex } \\
\text { exchange): } 100 \%\end{array}$ & $21 \%$ & $\mathrm{n} / \mathrm{a}$ & $\mathrm{n} / \mathrm{a}$ & $\begin{array}{l}\text { Inconsistent condom use during: } \\
\text { Vaginal sex }(74 \%) \\
\text { Oral sex }(83 \%) \\
\text { Anal sex }(18 \%)\end{array}$ \\
\hline $\begin{array}{l}\text { Alary et al. } \\
\text { (1994) }\end{array}$ & 48 & IDU-FSWs: $100 \%$ & $0 \%$ & $\begin{array}{l}\text { n/a } \\
\text { (Syphilis } \quad 0 \% \text {; } \\
\text { Gonorrhea } 3 \% \text {; } \\
\text { Genital } \\
\text { chlamydia } \\
\text { Hepatitis } \\
0.4 \% ; \quad \text { Benital } \\
\text { herpes } 2.2 \% \text {; } \\
\text { Vaginal } \\
\text { trichomoniasis } \\
\text { 7\%; Genital } \\
\text { warts } 4.2 \% \text {; any } \\
\text { STD 19\% } \\
\text { among all FSWs) }\end{array}$ & Needle sharing & $\begin{array}{l} \\
\text { Inconsistent condom use, w/ casual } \\
\text { non-paying partners: } \\
\text { Vaginal sex (74.6\%) } \\
\text { Oral sex (75.4\%) } \\
\text { (Street work) }\end{array}$ \\
\hline
\end{tabular}




\begin{tabular}{|c|c|c|c|c|c|c|}
\hline $\begin{array}{l}\text { Astemborski } \\
\text { \& Solomon } \\
\text { (1994) }\end{array}$ & 52 & $\begin{array}{l}\text { IDU-FSWs (sex trade) } \\
100 \%\end{array}$ & $\begin{array}{r}23.7 \% \quad \text { (low } \\
\text { traders) } \\
47.6 \% \quad \text { (high } \\
\text { traders) }\end{array}$ & $\mathrm{n} / \mathrm{a}$ & $\mathrm{n} / \mathrm{a}$ & $\begin{array}{l}\text { High sex trade }(>=50)(19.9 \%) \\
\text { IDU partners, }>2(52.1 \%)\end{array}$ \\
\hline $\begin{array}{l}\text { Taylor et al. } \\
\text { (1993) }\end{array}$ & 50 & IDU-FSWs: $100 \%$ & $2.2 \%$ & $\mathrm{n} / \mathrm{a}$ & $\begin{array}{l}\text { Sharing } \\
\text { needles/syringes, } 6 \\
\text { mos. prior to } \\
\text { study }(45 \%)\end{array}$ & $\begin{array}{l}\text { Inconsistent condom use w/ } \\
\text { non-paying primary partners }(91 \%) \\
\text { Inconsistent condom use w/ casual } \\
\text { partners }(78 \%) \\
\text { IDU partners }(76 \%)\end{array}$ \\
\hline $\begin{array}{l}\text { van den } \\
\text { Hoek et al. } \\
(1990)\end{array}$ & 95 & IDU-FSWs: $100 \%$ & $\begin{array}{l}\mathrm{n} / \mathrm{a} \\
(43 \% \quad \text { among } \\
\text { female IDUs })\end{array}$ & $\begin{array}{l}\text { Syphilis } 17 \% \text {; } \\
\text { Gonorrhea } \\
65 \% \text {; } \\
\text { Genital herpes } \\
24 \%\end{array}$ & $\mathrm{n} / \mathrm{a}$ & $\begin{array}{l}\text { Unprotected sex w/ customers: } \\
\text { Vaginal contact }(14 \%) \\
\text { Orogenital contact }(37 \%)\end{array}$ \\
\hline $\begin{array}{l}\text { van den } \\
\text { Hoek et al. } \\
\text { (1989) }\end{array}$ & 49 & $\begin{array}{l}\text { IDU-FSWs: } 82.1 \% \\
\text { NIDU-FSWs: } 17.9 \%\end{array}$ & $\begin{array}{l}30 \% \\
\text { (HIV antibody) }\end{array}$ & $\begin{array}{l}\text { Hepatitis B: } \\
67 \% \\
\text { Syphilis: } 20 \% \\
\text { (Gonorrhea } \\
\text { treatment } \\
\text { history: } 64 \% \text { ) }\end{array}$ & $\operatorname{IDU}(82 \%)$ & $\begin{array}{l}\text { Duration of sex work, }>=5 \text { yrs. }(55 \%) \\
\text { Frequency of gonorrhea, previous } 5 \\
\text { yrs. }(64 \%)\end{array}$ \\
\hline $\begin{array}{l}\text { Tirelli et al. } \\
\text { (1989) }\end{array}$ & 62 & IDU-FSWs: $100 \%$ & 36\% (IDU-FSWs) & $\mathrm{n} / \mathrm{a}$ & IDU $(100 \%)$ & $\begin{array}{l}\text { Unprotected sex (n/a) } \\
\text { IDU partner (n/a) }\end{array}$ \\
\hline
\end{tabular}

As to unprotected sex (e.g., no or inconsistent condom use), the most commonly examined sexual related risk factor, reviewed studies revealed several general patterns. First, IDU-FSWs practiced more unprotected sex than NIDU-FSWs as measured by responses to both questions about their "last sex act" and questions about sexual activity "in the past month" ( $25.9 \%$ vs. $15.7 \%$ and $64.4 \%$ vs. $31.1 \%$ respectively). Second, three studies showed that DU-FSWs practiced unprotected sex most commonly in oral sex, less commonly in vaginal sex, and least frequently in anal sex (Inciardi 1995; Alary, Worm, Kvinesdal, 1994; van den Hoek et al., 1989). Particularly, the frequency of unprotected sex varied depending on kinds of sexual practices: generally unprotected sex $(31.1-89.5 \%$, mean $=66.4 \%$, median $=64.4 \%$, median $=64.4 \%, 7$ studies $)$, unprotected oral sex $(37-83 \%$, mean $=65.1 \%$, median $=75.4 \%$, 3 studies $)$, unprotected vaginal sex $(14-74.6 \%$, mean $=54.2 \%$, median $=74 \%, 3$ studies), unprotected anal sex (18\%). Finally, one study showed that DU-FSWs practiced unprotected sex most often with their husband/boyfriends (95\%), less often with regular clients $(86 \%)$, and the least with occasional clients (74.8\%) (Medhi et al., 2012). This is in line with another study, which demonstrated that unprotected sex with non-paying primary partners was higher (91\%) than with casual partners $(78 \%)$ in Glasgow, UK (Taylor et al., 1993).

A few studies examined other risky sexual practices, including: sex under the influence of drugs (31-95\%, mean $=63 \%, 2$ studies) and sexual/physical violence $(24-78 \%$, mean $=51 \%, 2$ studies). Other studies also looked at certain types of partners as risk factors: IDU-partners $(23.7-79 \%$, mean $=51.9 \%$, mean $=46.7 \%$, 6 studies $)$, DU-partners (84\%), gay/bisexual partners $(42 \%)$, male sex worker partners $(36 \%)$, FSW-partner (24\%), and HIV-infected partners (18\%). IDU-FSWs were more likely to have IDU partners (Spittal et al., 2003; Gollub et al., 1998; Astemborski et al., 1994). The length of sex work was another risk factor in five studies and the cut off points were between 5-10 years in the profession. One study added vaginal douching as a risk factor (Wang et al., 2009), and another pointed to intercourse during menstruation (Nguyen et al., 2004).

Physical and sexual violence, such as physical intimidation and rape, were commonly experienced risk factors 
among DU-FSWs. This phenomenon has been noted in the existing literature on victimization among FSWs. For instance, in a study of South African FSWs (Wechsberg, Luseno, Lam, 2005), 64\% experienced client-initiated violence, and the majority of violent clients were intoxicated on alcohol and other drugs. In Vancouver, Canada, 57\% of DU-FSWs who used illicit drugs (excluding marijuana) and engaged in street-level sex work experienced gender-based violence (Shannon et al., 2009). Over 40\% of street FSWs in Miami experienced violence from clients in the previous year; $24.9 \%$ were beaten, $12.9 \%$ were raped, and $13.8 \%$ were threatened with weapons (Surratt et al., 2004). Our reviewed articles also show the consistent violent culture among DU-FSWs. Drug use among both FSWs and their clients exacerbated gender-based violence, as drug use jeopardized the women's negotiating power in selecting clients and implementing safe sex practices (Parry et al., 2009; Strathdee, et al., 2011), placed them at a greater risk of HIV/STIs (Azim et al., 2006), and often made clients more violent due to their frustrations with impotence (Leggett 2001). Furthermore, compared to their peers who did not use drugs, DU-FSWs were more likely to experience psychological distress, which put the women at enhanced risk for HIV infection (Tang et al., 2014; Seth et al., 2009; Ulibarri, Strathdee, Patterson, 2010).

\subsubsection{HIV Transmission Mechanisms}

There is uncertainty and discrepancy concerning the mechanism of HIV transmission among DU-FSWs. Early studies suggested a pattern in high-income countries in North America and Europe where HIV among FSWs was transmitted mainly through IDU (van den Hoek et al., 1988; Vioque et al., 1998), whereas in low- and mid-income countries in Africa and Asia, heterosexual transmission of HIV infection was the primary transmission route among FSWs (McKeganey, 1994; Kreiss et al., 1986; Nzila et al., 1991). However, that understanding has been complicated by more recent studies pointing to dual transmission of HIV/STIs among DU-FSWs.

\subsubsection{Drug Injection Transmission}

Consistent with the pattern of the HIV epidemic, earlier studies identified injection drug use as the primary risk factor for HIV infection. Compared with low HIV prevalence among non-DU-FSWs, higher rates of HIV infections were found among IDU-FSWs or their sexual partners (McKeganey, 1994). The studies suggested a pattern indicating that the prevalence of HIV infection among FSWs in high-income countries-in Europe and North America - was closely associated with intravenous drug use and sharing of injection equipment (Spittal et al., 2003; Nguyen et al., 2004; Alary, Worm, \& Kvinesdal, 1994; van den Hoek et al., 1989; Taylor et al., 1993; Tirelli et al., 1989; Rhodes et al., 1994). For instance, in Pordenone province, Italy, sharing of syringes was the most important mechanism of HIV transmission; prevalence of HIV seropositivity among IDU-FSWs was comparable with that of female IDUs (58\% vs. 62\%, respectively) (Dan et al., 1987). In the UK, there were no significant differences in HIV prevalence rates or HIV risk behavior between female IDUs and IDU-FSWs (Rhodes et al., 1994). Thus, in these earlier studies among FSWs in high-income countries, the largest risk factor of HIV infection for FSWs appeared to be injection drug use, and sex work did not constitute an additional risk factor in the prevalence of HIV among female IDUs (Taylor et al., 1993; Gillies \& Carballo, 1990). However, two reviewed studies showed an exception to this pattern. In three U.S. cities (Miami, New York, San Francisco), injection status was not associated with HIV rates among commercial sex workers (Jones et al., 1998). In Baltimore, another U.S. city, frequent sex traders had much higher HIV prevalence $(47.6 \%)$ than low or non-traders among female DUs (23.7\% and 23.2\% respectively) (Astemborski et al., 1994).

\subsubsection{Heterosexual Transmission}

Until the late 1990s, HIV and other STIs among DU-FSWs in low- and mid-income countries were regarded as the result of sexual transmission rather than drug use (Nguyen et al., 2004). For instance, while HIV prevalence was high among DU-FSWs in countries such as Thailand, China, Myanmar, Vietnam, and Congo, few non-DU-FSWs were infected with HIV, and thus infection was regarded as being transmitted through sexual activities with drug-using partners (Nzila et al., 1991; Siraprapasiri et al., 1991; Prybylski, Alto, 1999).

However, recent empirical studies have shown that substance use-especially injection drug use-has increasingly become a primary risk factor among FSWs in many low- and mid-income countries as well as in high-income countries. For instance, street-based IDU-FSWs in Ho Chi Minh City, Vietnam, were at high-risk for HIV infection (16.3\%) (Nguyen et al., 2004). Over $80 \%$ of registered HIV cases in Russia have occurred among intravenous drug users (Benotsch et al., 2004). In Guangzhou, Kaiyuan, and Kunming in China, a large portion (12-49\%) of IDU-FSWs were HIV-positive (Poon et al., 2011; Wang et al., 2009; Chen et al., 2005; van den Hoek et al., 2001). Although sexual transmission has become a dominant mode of HIV transmission in China in recent years (Li, Li, Stanton 2010; Tang et al., 2014), illegal drug use — especially the injection of drugs — has also been increasingly linked to HIV infection among Chinese FSWs (Poon, et al., 2011; Wang et al., 2009; van den Hoek 2001). In Tijuana and Ciudad Juarez, Mexico, drug-using behaviors (injection drugs, drug use before/during sex) 
played a more important role than sexual behavior in syphilis transmission among drug-using FSWs (Loza et al., 2010). Recreational drug use, both injection and non-injection, has been considered to be the greatest risk factor for HIV acquisition among FSWs in China (Wang et al., 2009; Xu et al., 2008).

\subsubsection{Dual Transmissions}

Recent evidence from the U.S. and Canada highlighted the importance of examining the complex relationship between injection drug use and heterosexual transmission of HIV/AIDS, particularly among women (Strathdee et al., 2001; Spittal et al., 2003; Kral et al., 2001). For example, risky sexual behavior was closely associated with HIV-1 seroconversion in street-based IDU-FSWs (Kral et al., 2001). Based on the data from 2,945 women drug injectors and crack users in sixteen low- and four high-sero-prevalence sites, researchers found that injection drug use and exchanging sex for money both played a major role in the transmission of HIV infection in the U.S. (Tortu et al., 2000). One reviewed study showed an exception to this pattern; HIV prevalence did not differ between non-IDU-FSWs (29.2\%) and IDU-FSWs (29\%) in Vancouver and Montreal, Canada (Spittal et al., 2003). Another study also showed an inconsistent relationship between drug use and HIV prevalence in the U.K.; IDU-FSWs had a $12.9 \%$ HIV rate whereas female IDUs not engaging in sex work had 14.4\% HIV prevalence (Rhodes et al., 1994); possible mechanisms through which DU-FSWs face a higher risk of HIV/STI might have been involved in both drug use and the sex trade, which are closely intertwined.

\subsubsection{Synergetic Effect Between the Sex Trade and Drug Use}

DU-FSWs are not merely at combined risk for each of the two risk behaviors but are at enhanced risk caused by synergetic effects between the two- a synergetic interaction between sex and drug behaviors that increased the overall HIV/STI risks beyond the two alone. IDU-FSWs tended to engage in more risky practices than their counterparts. For example, in Tijuana and Ciudad Juarez, Mexico, HIV prevalence among IDU-FSWs was more than twice that of FSWs who did not inject drugs (12\% versus 5\%) (Strathdee et al., 2008). The IDU-FSWs tended to show higher rates of borrowing injection equipment, practicing needle or syringe sharing (possibly with multiple sex partners), injecting with a needle/syringe previously used by someone known to be HIV/HCV positive, overdosing, and injecting on a daily basis (Platt et al., 2005; Gu et al., 2008; Weber et al., 2002; Spittal et al., 2003). IDU-FSWs often shared needles with their IDU clients if the clients sent them to procure drugs and the needles to use, therefore borrowing and lending equipment was often a part of the sex trade (Spittal et al., 2003; Spittal et al., 2001).

Only recently have scholars investigated the overlap between drug use and sexual transmission among FSWs (Medhi et al., 2012; Cusick 2006; Hong, Xu, Zhang 2013). Drug use was closely connected to sexual risk behavior, as sexual practice was often influenced by drug use or addiction. Drug users were more sexually active and more likely to practice unsafe sex, such as having more sexual partners, using condoms less regularly, and beginning sexual activity at an earlier age (CDCP 2005; DiClemente et al., 2001; Whitaker, Miller, 2000). For example, female street youth (aged 14-25 years) involved in sex work in Montreal, Canada were more likely to have reported a history of injection drug use (65\%) compared to girls with no history in the sex trade (33\%) (Weber et al., 2002). Among female heroin users in Gansu, China, injection drug use was independently associated with sex work; furthermore, unsafe drug use practices, such as injection and needle sharing, were more common among heroin-using FSWs than other female heroin users (Wang, Lin, 2003).

Additionally, the use of recreational drugs (including ecstasy, ketamine, and methamphetamine) and drug use during sexual contact(s) impairs judgment, negatively impacting the ability to negotiate safe sex (Chen et al., 2012; Parry et al., 2009; Hong, Xu, Zhang, 2013; Lomba, Apostolo, Mendes, 2009; Hayaki, Anderson, Stein, 2006). In nightclub settings, nearly half of Portuguese youth (46.7\%) practiced unsafe sex under the influence of recreational drugs (Lomba, Apostolo, Mendes, 2009). FSWs under the influence of crack may also be less careful when choosing sexual practices or partners (Inciardi 1995; Jones et al., 1998). Furthermore, substance use enhanced sexual experiences and prolonged sex sessions (Parry et al., 2009), and unprotected sex was common after injecting drugs or other drug use such as ecstasy, ketamine, and methamphetamine (Hong, Xu, Zhang, 2013). An exception was among FSWs in south London where researchers found no overall association between any of the drug use variables and the likelihood of unprotected sex (Gossop et al., 1995).

\section{Discussion}

Our review synthesized the existing literature and found that DU-FSWs were at significantly higher risk for HIV/STI and displayed more risk behaviors than other women (i.e., non-DU-FSWs, non-sex-worker female DUs, general female population), suggesting a synergy effect of drug use and sex work. By consolidating the major findings, we intended to identify the knowledge gaps in the field and discuss the direction of future research. To the 
best of our knowledge, this is the first systematic review of global literature on drug-using FSWs, and it provided potential directions for future research. The existing literature underscored the vulnerability of DU-FSWs regarding HIV/STI status, despite the variation in the extent of the prevalence, geographic locations, socio-cultural context, and characteristics of the study community. Still, much remains to be examined regarding the vulnerable, and often stigmatized groups of women who are at doubled risk. In particular, further research is critical to more fully understand the synergetic relationship between drug use and sex work.

While earlier studies pointed to a general pattern of drug using being a single primary risk factor for DU-FSWs, findings in current research have suggested that both sexual risk behaviors and drug-related behavior are important risk factors for transmitting HIV/STIs and that the two factors are closely intertwined. Recent empirical studies from both low- and mid-income countries and high-income countries present a complex picture of the relationship between drug use and HIV/STI status among FSWs. A greater number of FSWs in low- and mid-income countries are increasingly using various illicit drugs, as are their counterparts in high-income countries, which is becoming a major risk factor for HIV/STI transmission.

The variation in findings regarding the direction and magnitude of the associations may result from the limited number of studies targeting drug-using FSWs, as DU-FSWs were often examined as a sub-population along with other high-risk groups. The complexity may also result from multiple dimensions of the mechanisms behind HIV-related risk. The reviewed studies suggested that the effects of drug use and the sex trade vary with a number of social context factors and specificities of the study population, which are important influencing factors, though not risk factors themselves.

First, gender disparity significantly affects the risk of HIV infection through economic and social pressures that enhance exposure to risk (Davies et al., 1996) by negatively influencing women's capacity to negotiate safer sexual and injection practices (Platt et al., 2005). DU-FSWs are compelled to maintain higher volumes of clients to fund their drug habits, thus increasing their exposure to HIV and other STIs, and they are more likely to agree to forego condom use for more money or in order to secure clients (Leggett, 2001; Parry et al., 2009). Due to economic deprivation and lack of empowerment, DU-FSWs are more likely to practice unprotected sex for more money or accept clients who have a higher likelihood of HIV infection (Tang et al., 2014; Zhang et al., 2013). In addition, because of heavy economic pressure, many drug users chose injection and preferred to share and reuse needles; both practices precipitated the risk of HIV infection (Choi, Cheung, \& Chen, 2006).

Second, the lack of social support is another important risk factor for HIV/STI. In most countries, HIV prevalence among drug users including IDU-FSWs is higher not only because they engage in risky behaviors but also because they are significantly stigmatized and subject to discrimination, and because they often lack access to healthcare services (Malta et al., 2008; Parry et al., 2009; UNAIDS 2006). The combined problems of poor social support, homelessness, living in slums, and limited access to health care place drug-using sex workers at a greater risk for HIV/STIs (Parry et al., 2009).

Third, the potential mechanisms of HIV/STI transmission are also associated with a particular social context - including cultural perception of stigma, policing practices, and public policy (e.g., needle policy), which, when combined, compromise the women's physical and psychological well-being. Women's social environments, rather than individual-level risk behaviors, may determine the risk factors associated with risk of HIV infection. For instance, in northern Mexico, police confiscation of syringes in lieu of arrest and needle exchange programs (NEPs) led DU-FSWs to share needles more often, which was the primary risk factor for HIV infection among the women (Strathdee et al., 2011). Another study in the U.K. also indicated the importance of policy-level factors in HIV transmission; the low rate of HIV infection among the street-working IDU-FSWs may be because sterile injection equipment was provided by a street-level, drop-in clinic within the red-light area and the network of needle exchange clinics (McKeganey et al., 1992). In South Africa, cultural beliefs opposing condom use were found to negatively affect FSWs' ability to sustain safer sex behaviors (Leggett, 2001; Parry, 2009; Wojcicki \& Malala, 2001). HIV prevalence also varied depending on the sex venue (Chen et al., 2005). Lower-end venues such as streets had the highest prevalence of HIV infection and higher-end venues such as nightclubs had the lowest (Wang et al., 2009; Alary, Worm, \& Kvinesdal, 1994).

Of the multiple risk factors involved in the transmission dynamics of HIV among DU-FSWs, individual risk factors have been the most commonly examined. However, few empirical studies have been conducted to investigate network and structural factors that may increase or reduce the level of risk. Such network features (e.g., dynamics of various relationships, characteristics of partners, network properties such as density and size) as well as socio-economic structural factors (e.g., access to health care, physical violence, police confiscation, welfare policies) appear to be influential risk determinants of the current differential incidence rates of HIV and other STIs 
in vulnerable populations. For instance, DU-FSWs were at high risk for HIV as a result of their relationships with their primary sex partners, who were often IDUs (Miller \& Neaigus, 2001). Social factors such as homelessness and imprisonment (Malta et al., 2008) might be as significant as individual-level risk behavior among DU-FSWs. In examining risk factors, future research needs to focus more on the interpersonal, network, and structural dimensions of DU-FSWs' experiences to better understand HIV transmission dynamics.

There were some limitations in the current literature review. First, non-English-language articles, gray literature, or unpublished studies (e.g., dissertations) were not included in this review because of concerns regarding the accessibility of these studies for a general audience. Second, the current review was largely focused on the issues of HIV-related risks but not on HIV treatment and care. Third, we did not perform meta-analysis to calculate and compare effective size of the selected studies because of the large variation or inconsistency in both STIs and risk factors in the existing studies. Finally, some data were old and did not reflect the HIV epidemic in recent years.

Despite these limitations, the findings of the current review have several important implications for future research and interventions. While an increasing number of empirical studies on drug-using FSWs and HIV/STI-related risk indicated the importance of HIV prevention and intervention for DU-FSWs, the findings about the association between drug use and the rates of HIV/STIs among FSWs varied across diverse cultural contexts. In order to identify the effects of sexual and drug-related risk behaviors relevant to the mechanisms of HIV/STI infection, researchers and health professionals need also to consider the social context of targeted populations, law enforcement in their communities, and complicated power dynamics within social and gender relations.

Future research also needs to consider a number of issues related to research methodology. First, future studies should be guided by theoretical frameworks that take into account the pathways underlying the association between drug use and HIV/STIs among FSWs. For example, we need to develop a conceptual framework, using both qualitative and quantitative methods, regarding the synergetic HIV risk between drug use and the sex trade. Among the reviewed studies, only one qualitative study offered a conceptual model with qualitative data (Malta et al., 2008). Future studies should likewise employ a longitudinal study design whenever possible in order to establish a meaningful causal relationship between the use of drugs and HIV prevalence among FSWs, and recruit an adequate number of participants to ensure the power of analysis. Finally, more qualitative studies need to be conducted in order to identify the synergetic dynamics between sex work and drug use, as they will yield useful insights for future interventions and study design.

We suggest potential directions for future research on DU-FSWs. The first possibility would be to identify the drug use networks and sexual networks among DU-FSWs, to examine how these networks overlap, and to determine whether the overlap is associated with HIV-related risk. We hypothesize that the great variation in HIV risk among DU-FSWs in various settings may be the result of the level of the overlap between drug use networks and sex work networks. By taking this direction, we could contribute to the development of prevention theory by constructing risk profiles and comparing the roles of these networks in HIV-related risks among DU-FSWs. In addition, by identifying the subgroup of FSWs who are at particularly high risk of HIV infection, this approach may improve HIV/STI intervention practices. Methodologically, we could apply mobile tools (e.g., use of online RDS for recruitment, use of iPads or smart phones) to identify DU-FSWs, an often a hard-to-reach population. This may contribute to methodological innovations to collect social network data among hidden populations. Furthermore, we need to examine possible pharmacological and biological mechanisms behind the synergetic effects between drug use and sex work. Lastly, when targeting DU-FSWS with multiple risk behaviors, health professionals and institutions should employ HIV prevention strategies that combine behavioral, biomedical, and structural approaches.

\section{Funding}

The Eunice Kennedy Shriver National Institute of Child Health and Human Development (NICHD) US National Institutes of Health (NIH) Research Grant (R01HD074221) supported the study. The Western Washington University (WWU) College of Humanities \& Social Sciences (CHSS) Summer Grant supported the revision of this article. The content is the sole responsibility of the authors and does not necessarily represent the offcial views of the NIH/NICHD or WWU CHSS.

\section{Competing Interests Statement}

The authors declare that there are no competing or potential conflicts of interest.

\section{References}

Alary, M., Worm, A. M., \& Kvinesdal, B. (1994). Risk Behaviors for HIV-infection and Sexually-transmitted diseases among Female Sex Workers from Copenhagen. Int $J$ STD AIDS, 5(5), 365-367. 
https://doi.org/10.1177/095646249400500516

Astemborski, J., Vlahov, D., Warren, D., Solomon, L., \& Nelson, K. E. (1994). The trading of sex for drugs or money and HIV seropositivity among female intravenous drug users. Am J Public Health, 84(3), 382-387. https://doi.org/10.2105/AJPH.84.3.382

Azim, T., Chowdhury, E. I., Reza, M., Ahmed, M., Uddin, M. T., Khan, R., ... \& Sack, D. A. (2006). Vulnerability to HIV infection among sex worker and non-sex worker female injecting drug users in Dhaka, Bangladesh: evidence from the baseline survey of a cohort study. Harm Reduction Journal, 3(1), 33. https://doi.org/10.1186/1477-7517-3-33

Baral, S., Todd, C. S., Aumakhan, B., Lloyd, J., Delegchoimbol, A., \& Sabin, K. (2013). HIV among female sex workers in the Central Asian Republics, Afghanistan, and Mongolia: contexts and convergence with drug use. Drug Alcohol Depend, 132(Suppl 1), S13-16. https://doi.org/10.1016/j.drugalcdep.2013.07.004

Bautista, C. T., Sanchez, J. L., Montano, S. M., Laguna-Torres, A., Suarez, L., Sanchez, J., ... \& Aguayo, N. (2006). Seroprevalence of and risk factors for HIV-1 infection among female commercial sex workers in South America. Sexually transmitted infections, 82(4), 311-316. https://doi.org/10.1136/sti.2005.018234

Belza, M. J., \& Spanish Grp Unlinked Anonymous, S. (2004). Prevalence of HIV, HTLV-I and HTLV-II among female sex workers in Spain, 2000-2001. European Journal of Epidemiology, 19(3), 279-282. https://doi.org/10.1023/B:EJEP.0000020443.29480.d0

Benotsch, E. G., Somlai, A. M., Pinkerton, S. D., Kelly, J. A., Ostrovski, D., Gore-Felton, C., \& Kozlov, A. P. (2004). Drug use and sexual risk behaviours among female Russian IDUs who exchange sex for money or drugs. International journal of STD \& AIDS, 15(5), 343-347. https://doi.org/10.1177/095646240401500514

Boily, M. C., Baggaley, R. F., Wang, L., Masse, B., White, R. G., Hayes, R. J., \& Alary, M. (2009). Heterosexual risk of HIV-1 infection per sexual act: systematic review and meta-analysis of observational studies. The Lancet infectious diseases, 9(2), 118-129. https://doi.org/10.1016/S1473-3099(09)70021-0

Centers for Disease Control and Prevention. (2006). Youth risk behavior surveillance-United States, 2005. Morbidity and Mortality Weekly Report, 55SS-5.

Chen, X. S., Wang, Q. Q., Yin, Y. P., Liang, G. J., Jiang, N., Yang, L. G., ... \& Wang, B. (2012). Prevalence of syphilis infection in different tiers of female sex workers in China: implications for surveillance and interventions. BMC infectious diseases, 12(1), 84. https://doi.org/10.1186/1471-2334-12-84

Chen, X. S., Yin, Y. P., Liang, G. J., Gong, X. D., Li, H. S., Poumerol, G., ... \& Yu, Y. H. (2005). Sexually transmitted infections among female sex workers in Yunnan, China. AIDS Patient Care \& STDs, 19(12), 853-860. https://doi.org/10.1089/apc.2005.19.853

CHLP (The Center for HIV Law and Policy). (2015). Routes, Risks and Realities of HIV Transmission and Care: Current Scientic Knowledge and Medical Treatment.

Choi, S. Y., Cheung, Y. W., \& Chen, K. (2006). Gender and HIV risk behavior among intravenous drug users in Sichuan Province. China. Soc Sci Med, 62(7), 1672-1684. https://doi.org/10.1016/j.socscimed.2005.08.046

Cusick, L. (2006). Widening the harm reduction agenda: From drug use to sex work. International Journal of Drug Policy, 17(1), 3-11. https://doi.org/10.1016/j.drugpo.2005.12.002

Dan, M., Bar-Shani, S., Tirelli, U., \& Rock, M. (1987). HIV antibodies in drug-addicted prostitutes. JAMA, 257(8), 1047-1047. https://doi.org/10.1001/jama.1987.03390080037011

Davies, A. G., Dominy, N. J., Peters, A. D., \& Richardson, A. M. (1996). Gender differences in HIV risk behaviour of injecting drug users in Edinburgh. AIDS Care, 8(5), 517-527. https://doi.org/10.1080/09540129650125489

de Graaf, R., Vanwesenbeeck, I., van Zessen, G., Straver, C. J., \& Visser, J. H. (1995). Alcohol and drug use in heterosexual and homosexual prostitution, and its relation to protection behaviour. AIDS Care, 7(1), 35-47. https://doi.org/10.1080/09540129550126948

Dehne, K., \& Kobyshcha, Y. (2000). The HIV epidemic in central and eastern Europe: update 2000.

DiClemente, R. J., Wingood, G. M., Crosby, R., Cobb, B. K., Harrington, K., \& Davies, S. L. (2001). Parent-adolescent communication and sexual risk behaviors among African American adolescent females. $J$ Pediatr, 139(3), 407-412. https://doi.org/10.1067/mpd.2001.117075

Dunne, E. M., Dyer, T. P., Khan, M. R., Cavanaugh, C. E., Melnikov, A., \& Latimer, W. W. (2014). HIV prevalence and risk behaviors among African American Women Who Trade Sex for Drugs Versus Economic Resources. 
AIDS Behav, 18(7), 1288-1292. https://doi.org/10.1007/s10461-014-0710-6

Edlin, B. R., Irwin, K. L., Faruque, S., McCoy, C. B., Word, C., Serrano, Y., ... \& Multicenter Crack Cocaine and HIV Infection Study Team. (1994). Intersecting epidemics--crack cocaine use and HIV infection among inner-city young adults. New England Journal of Medicine, 331(21), 1422-1427. https://doi.org/10.1056/NEJM199411243312106

Estébanez, P., Zunzunegui, M. V., Aguilar, M. D., Coloma, C., RUA-FIGUEROA, M. I. L. A. G. R. O. S. A., Fitch, K., ... \& Nájera, R. (1998). A demographic and health survey of Spanish female sex workers: HIV prevalence and associated risk factors. Journal of biosocial science, 30(3), 365-379. https://doi.org/10.1017/S0021932098003654

Gillies, P., \& Carballo, M. (1990). Adult perception of risk, risk behaviour and HIV/AIDS: a focus for intervention and research. Aids, 4(10), 943-952. https://doi.org/10.1097/00002030-199010000-00001

Gollub, E. L., Rey, D., Obadia, Y., \& Moatti, J. P. (1998). Gender differences in risk behaviors among HIV+ persons with an IDU history. The link between partner characteristics and women's higher drug-sex risks. The $\begin{array}{llllll}\text { Manif } 2000 \quad \text { Study } & \text { Group. Sex } & \text { Transm }\end{array}$ https://doi.org/10.1097/00007435-199810000-00008

Gossop, M., Powis, B., Griffiths, P., \& Strang, J. (1994). Sexual behaviour and its relationship to drug-taking among prostitutes in south London. Addiction, 89(8), 961-970. https://doi.org/10.1111/j.1360-0443.1994.tb03356.x

Gossop, M., Powis, B., Griffiths, P., \& Strang, J. (1995). Female prostitutes in south London: use of heroin, cocaine and alcohol, and their relationship to health risk behaviours. AIDS Care, 7(3), 253-260. https://doi.org/10.1080/09540129550126498

Gu, J., Chen, H., Chen, X., Lau, J. T., Wang, R., Liu, C., ... \& Li, Z. (2008). Severity of drug dependence, economic pressure and HIV-related risk behaviors among non-institutionalized female injecting drug users who are also sex workers in China. Drug \& Alcohol Dependence, 97(3), 257-267. https://doi.org/10.1016/j.drugalcdep.2008.03.029

Hayaki, J., Anderson, B., \& Stein, M. (2006). Sexual risk behaviors among substance users: relationship to impulsivity. Psychol Addict Behav, 20(3), 328-332. https://doi.org/10.1037/0893-164X.20.3.328

Hong, H., Xu, G. Z., \& Zhang, D. D. (2013). HIV Risk, Drug Use, and Sexual transmission among Female Sex Workers in Ningbo, China. Int J Gynaecol Obstet, 121(1), 91-92. https://doi.org/10.1016/j.ijgo.2012.11.004

Inciardi, J. A., Surratt, H. L., \& Kurtz, S. P. (2006). HIV, HBV, and HCV infections among drug-involved, inner-city, street sex workers in Miami, Florida. AIDS Behav, 10(2), 139-147. https://doi.org/10.1007/s10461-005-9049-3

Inciardi, J. A. (1995). Crack, Crack house sex, and HIV risk. Arch Sex Behav, 24(3), 249-269. https://doi.org/10.1007/BF01541599

Jones, D. L., Irwin, K. L., Inciardi, J., Bowser, B., Schilling, R., Word, C., ... \& Edlin, B. R. (1998). The high - risk sexual practices of crack - smoking sex workers recruited from the streets of three American cities. Sexually Transmitted Diseases, 25(4), 187-193. https://doi.org/10.1097/00007435-199804000-00002

Konings, E. (1996). Prostitution and HIV/AIDS in CEE/CIS. Geneva, Switzerland: UNAIDS.

Kral, A. H., Bluthenthal, R. N., Lorvick, J., Gee, L., Bacchetti, P., \& Edlin, B. R. (2001). Sexual transmission of HIV-1 among injection drug users in San Francisco, USA: risk-factor analysis. Lancet, 357(9266), 1397-1401. https://doi.org/10.1016/S0140-6736(00)04562-1

Kreiss, J. K., Koech, D., Plummer, F. A., Holmes, K. K., Lightfoote, M., Piot, P., ... \& Ngugi, E. N. (1986). AIDS virus infection in Nairobi prostitutes. New England journal of medicine, 314(7), 414-418. https://doi.org/10.1056/NEJM198602133140704

Kwiatkowski, C. F., \& Booth, R. E. (2000). Differences in HIV risk behaviors among women who exchange sex for drugs, money, or both drugs and money. AIDS and Behavior, 4(3), 233-240. https://doi.org/10.1023/A:1009512601057

Lau, J. T., Tsui, H. Y., Zhang, Y., Cheng, F., Zhang, L., Zhang, J., \& Wang, N. (2008). Comparing HIV-related syringe-sharing behaviors among female IDU engaging versus not engaging in commercial sex. Drug Alcohol Depend, 97(1-2), 54-63. https://doi.org/10.1016/j.drugalcdep.2008.03.024 
Lau, J. T., Zhang, J., Zhang, L., Wang, N., Cheng, F., Zhang, Y., ... \& Lan, Y. (2007). Comparing prevalence of condom use among 15,379 female sex workers injecting or not injecting drugs in China. Sexually transmitted diseases, 34(11), 908-916. https://doi.org/10.1097/OLQ.0b013e3180e904b4

Leggett, T. (2001). Drugs, sex work, and HIV in three South African cities. Society in Transition, 32(1), 101-109. https://doi.org/10.1080/21528586.2001.10419034

Li, Q., Li, X., \& Stanton, B. (2010). Alcohol use among female sex workers and male clients: an integrative review of global literature. Alcohol and Alcoholism, 45(2), 188-199. https://doi.org/10.1093/alcalc/agp095

Logan, T. K., Leukefeld, C., \& Farabee, D. (1998). Sexual and drug use behaviors among women crack users: implications for prevention. AIDS Educ Prev, 10(4), 327-340.

Logan, T. K., \& Leukefeld, C. (2000). Sexual and drug use behaviors among female crack users: a multi-site sample. Drug Alcohol Depend, 58(3), 237-245. https://doi.org/10.1016/S0376-8716(99)00096-4

Lomba, L., Apostolo, J., \& Mendes, F. (2009). Drugs and alcohol consumption and sexual behaviours in night recreational settings in Portugal. Adicciones, 21(4), 309-325. https://doi.org/10.20882/adicciones.222

Loza, O., Patterson, T. L., Rusch, M., Martínez, G. A., Lozada, R., Staines - Orozco, H., ... \& Strathdee, S. A. (2010). Drug - related behaviors independently associated with syphilis infection among female sex workers in two Mexico-US border cities. Addiction, 105(8), 1448-1456. https://doi.org/10.1111/j.1360-0443.2010.02985.x

Malta, M., Monteiro, S., Lima, R. M. J., Bauken, S., Marco, A. D., Zuim, G. C., ... \& Strathdee, S. A. (2008). HIV/AIDS risk among female sex workers who use crack in Southern Brazil. Revista de saúde pública, 42(5), 830-837. https://doi.org/10.1590/S0034-89102008000500007

MAPN (The Monitoring the AIDS Pandemic Network. (2005). Drug injection and HIV/AIDS in Asia. The MAP Report.

McKeganey, N., Barnard, M., Leyland, A., Coote, I., \& Follet, E. (1992). Female streetworking prostitution and HIV infection in Glasgow. Bmj, 305(6857), 801-804. https://doi.org/10.1136/bmj.305.6857.801

McKeganey, N. P. (1994). Prostitution and HIV: what do we know and where might research be targeted in the future? AIDS, 8(9), 1215-1226. https://doi.org/10.1097/00002030-199409000-00002

Medhi, G. K., Mahanta, J., Kermode, M., Paranjape, R. S., Adhikary, R., Phukan, S. K., \& Ngully, P. (2012). Factors associated with history of drug use among female sex workers (FSW) in a high HIV prevalence state of India. BMC Public Health, 12, 273. https://doi.org/10.1186/1471-2458-12-273

Miller, M., \& Neaigus, A. (2001). Networks, resources and risk among women who use drugs. Soc Sci Med, 52(6), 967-978. https://doi.org/10.1016/S0277-9536(00)00199-4

Tuan, N. A., Hien, N. T., Chi, P. K., Thang, B. D., Long, H. T., Saidel, T., \& Detels, R. (2004). Intravenous drug use among street-based sex workers: a high-risk behavior for HIV transmission. Sexually transmitted diseases, 31(1), 15-19. https://doi.org/10.1097/01.OLQ.0000105002.34902.B5

Nzila, N., Laga, M., Thiam, M. A., Mayimona, K., Edidi, B., Van Dyck, E., ... \& Ashley, R. L. (1991). HIV and other sexually transmitted diseases among female prostitutes in Kinshasa. Aids. https://doi.org/10.1097/00002030-199106000-00011

Panda, S., Bijaya, L., Devi, N. S., Foley, E., Chatterjee, A., Banerjee, D., ... \& Bhattacharya, S. K. (2001). Interface between drug use and sex work in Manipur. National Medical Journal of India, 14(4), 209-210.

Parry, C., Petersen, P., Carney, T., Dewing, S., \& Needle, R. (2009). Rapid assessment of drug use and sexual HIV risk patterns among vulnerable drug-using populations in Cape Town, Durban and Pretoria, South Africa. Sahara j, 5(3), 113-119. https://doi.org/10.1080/17290376.2008.9724909

Patterson, T. L., Semple, S. J., Staines, H., Lozada, R., Orozovich, P., Bucardo, J., ... \& Strathdee, S. A. (2008). Prevalence and correlates of HIV infection among female sex workers in 2 Mexico-US border cities. Journal of Infectious Diseases, 197(5), 728-732. https://doi.org/10.1086/527379

Platt, L., Rhodes, T., Lowndes, C. M., Madden, P., Sarang, A., Mikhailova, L., ... \& Khutorskoy, M. (2005). Impact of gender and sex work on sexual and injecting risk behaviors and their association with HIV positivity among injecting drug users in an HIV epidemic in Togliatti City, Russian Federation. Sexually transmitted diseases, 32(10), 605-612. https://doi.org/10.1097/01.olq.0000175391.13947.f7

Platt, L., Rhodes, T., Judd, A., Koshkina, E., Maksimova, S., Latishevskaya, N., ... \& Parry, J. V. (2007). Effects of 
sex work on the prevalence of syphilis among injection drug users in 3 Russian cities. American journal of public health, 97(3), 478-485. https://doi.org/10.2105/AJPH.2005.069732

Poon, A. N., Li, Z., Wang, N., \& Hong, Y. (2011). Review of HIV and other sexually transmitted infections among female sex workers in China. AIDS Care, 23(Suppl 1), 5-25. https://doi.org/10.1080/09540121.2011.554519

Prybylski, D., \& Alto, W. A. (1999). Knowledge, attitudes and practices concerning HIV/AIDS among sex workers in Phnom Penh, Cambodia. AIDS Care, 11(4), 459-472. https://doi.org/10.1080/09540129947857

Rhodes, T., Donoghoe, M., Hunter, G., \& Stimson, G. V. (1994). HIV prevalence no higher among female drug injectors also involved in prostitution. AIDS Care, 6(3), 269-276. https://doi.org/10.1080/09540129408258639

Seth, P., Raiji, P. T., DiClemente, R. J., Wingood, G. M., \& Rose, E. (2009). Psychological distress as a correlate of a biologically confirmed STI, risky sexual practices, self-efficacy and communication with male sex partners in African-American female adolescents. Psychol Health Med, 14(3), 291-300. https://doi.org/10.1080/13548500902730119

Shannon, K., Kerr, T., Strathdee, S. A., Shoveller, J., Montaner, J. S., \& Tyndall, M. W. (2009). Prevalence and structural correlates of gender based violence among a prospective cohort of female sex workers. Bmj, 339, b2939. https://doi.org/10.1136/bmj.b2939

Silbert, M. H., Pines, A. M., \& Lynch, T. (1982). Substance abuse and prostitution. J Psychoactive Drugs, 14(3), 193-197. https://doi.org/10.1080/02791072.1982.10471928

Siraprapasiri, T., Thanprasertsuk, S., Rodklay, A., Srivanichakorn, S., Sawanpanyalert, P., \& Temtanarak, J. (1991). Risk factors for HIV among prostitutes in Chiangmai, Thailand. AIDS, 5(5), 579-582. https://doi.org/10.1097/00002030-199105000-00017

Spittal, P. M., Bruneau, J., Craib, K. J., Miller, C., Lamothe, F., Weber, A. E., ... \& Schechter, M. T. (2003). Surviving the sex trade: a comparison of HIV risk behaviours among street-involved women in two Canadian cities who inject drugs. AIDS care, 15(2), 187-195. https://doi.org/10.1080/0954012031000068335

Strathdee, S. A., Lozada, R., Martinez, G., Vera, A., Rusch, M., Nguyen, L., ... \& Patterson, T. L. (2011). Social and structural factors associated with HIV infection among female sex workers who inject drugs in the Mexico-US border region. PloS one, 6(4), e19048.

Strathdee, S. A., Galai, N., Safaiean, M., Celentano, D. D., Vlahov, D., Johnson, L., \& Nelson, K. E. (2001). Sex differences in risk factors for hiv seroconversion among injection drug users: a 10-year perspective. Arch Intern Med, 161(10), 1281-1288. https://doi.org/10.1001/archinte.161.10.1281

Surratt, H. L., Inciardi, J. A., Kurtz, S. P., \& Kiley, M. C. (2004). Sex work and drug use in a subculture of violence. Crime Delinquency, 50, 43-59. https://doi.org/10.1177/0011128703258875

Strathdee, S. A., Philbin, M. M., Semple, S. J., Pu, M., Orozovich, P., Martinez, G., . . Patterson, T. L. (2008). Correlates of injection drug use among female sex workers in two Mexico-U.S. border cities. Drug Alcohol Depend, 92(1-3), 132-140. https://doi.org/10.1016/j.drugalcdep.2007.07.001

Spittal, P. M., Laliberte, N., Brooks, R., Tyndall, M., O'Shaughnessy, M. V., \& Schechter, M. T. (2001). Love, intimacy, violence, and needles: exploring the socio-cultural and relational contexts of risk in female injection drug users. Can J Infect Dis, 12.

Szwarcwald, C. L., Bastos, F. I., Gravato, N., Lacerda, R., Chequer, P. N., \& de Castilho, E. A. (1998). The relationship of illicit drug use to HIV-infection among commercial sex workers in the city of Santos, São Paulo, Brazil. International Journal of Drug Policy, 9(6), 427-436. https://doi.org/10.1016/S0955-3959(98)00059-0

Tang, Z., Zhang, C., Li, X., Liu, Y., Su, S., Zhou, Y., \& Shen, Z. (2014). HIV risk among female sex workers with different patterns of drug use behaviors in Southwest China: a cross-sectional study. AIDS Care, 1-8.

Taylor, A., Frischer, M., McKeganey, N., Goldberg, D., Green, S., \& Platt, S. (1993). HIV risk behaviours among female prostitute drug injectors in Glasgow. Addiction, 88(11), 1561-1564. https://doi.org/10.1111/j.1360-0443.1993.tb03142.x

Tirelli, U., Rezza, G., Giuliani, M., Caprilli, F., Gentili, G., Lazzarin, A., DeMercato, R. (1989). HIV seroprevalence among 304 female prostitutes from four Italian towns. Aids, 3(8), 547-548. 
https://doi.org/10.1097/00002030-198908000-00015

Tortu, S., Beardsley, M., Deren, S., Williams, M., McCoy, H. V., Stark, M., . . Goldstein, M. (2000). HIV infection and patterns of risk among women drug injectors and crack users in low and high sero-prevalence sites. AIDS Care, 12(1), 65-76. https://doi.org/10.1080/09540120047486

Tran, T. N., Detels, R., Long, H. T., Van Phung, L., \& Lan, H. P. (2005). HIV infection and risk characteristics among female sex workers in Hanoi, Vietnam. J Acquir Immune Defic Syndr, 39(5), 581-586.

Tyndall, M. W., Patrick, D., Spittal, P., Li, K., O'shaughnessy, M. V., \& Schechter, M. T. (2002). Risky sexual behaviours among injection drugs users with high HIV prevalence: implications for STD control. Sexually Transmitted Infections, 78(suppl 1), i170-i175. https://doi.org/10.1136/sti.78.suppl_1.i170

Ulibarri, M. D., Strathdee, S. A., \& Patterson, T. L. (2010). Sexual and drug use behaviors associated with HIV and other sexually transmitted infections among female sex workers in the Mexico-US border region. Curr Opin Psychiatry, 23(3), 215-220. https://doi.org/10.1097/YCO.0b013e32833864d5

United Nations \& AIDS (UNAIDS), 2006. Report on the global AIDS epidemic. A UNAIDS 10th anniversary special edition.

van den Hoek, A., Yuliang, F., Dukers, N. H., Zhiheng, C., Jiangting, F., Lina, Z., \& Xiuxing, Z. (2001). High prevalence of syphilis and other sexually transmitted diseases among sex workers in China: potential for fast spread of HIV. Aids, 15(6), 753-759. https://doi.org/10.1097/00002030-200104130-00011

van den Hoek, J. A., van Haastrecht, H. J., Scheeringa-Troost, B., Goudsmit, J., \& Coutinho, R. A. (1989). HIV infection and STD in drug addicted prostitutes in Amsterdam: potential for heterosexual HIV transmission. Genitourin Med, 65(3), 146-150. https://doi.org/10.1136/sti.65.3.146

van den Hoek, A., van Haastrecht, H. J., \& Coutinho, R. A. (1990). Heterosexual behaviour of intravenous drug users in Amsterdam: implications for the AIDS epidemic. Aids, 4(5), 449-453. https://doi.org/10.1097/00002030-199005000-00011

van den Hoek, J. A., Coutinho, R. A., van Haastrecht, H. J., van Zadelhoff, A. W., \& Goudsmit, J. (1988). Prevalence and risk factors of HIV infections among drug users and drug-using prostitutes in Amsterdam. Aids, 2(1), 55-60. https://doi.org/10.1097/00002030-198802000-00010

Vioque, J., Hernandez-Aguado, I., Fernandez Garcia, E., Garcia de la Hera, M., \& Alvarez-Dardet, C. (1998). Prospective cohort study of female sex workers and the risk of HIV infection in Alicante, Spain (1986-96). Sex Transm Infect, 74(4), 284-288. https://doi.org/10.1136/sti.74.4.284

Wang, H., Chen, R. Y., Ding, G., Ma, Y., Ma, J., Jiao, J. H., ... \& Wang, N. (2009). Prevalence and predictors of HIV infection among female sex workers in Kaiyuan City, Yunnan Province, China. International journal of infectious diseases, 13(2), 162-169. https://doi.org/10.1016/j.ijid.2008.05.1229

Wang, Q., \& Lin, G. (2003). Sex exchange and HIV-related risk behaviors among female heroin users in China. Journal of drug issues, 33(1), 119-132. https://doi.org/10.1177/002204260303300106

Weber, A. E., Boivin, J. F., Blais, L., Haley, N., \& Roy, E. (2002). HIV risk profile and prostitution among female street youths. J Urban Health, 79(4), 525-535. https://doi.org/10.1093/jurban/79.4.525

Weatherby, N. L., Shultz, J. M., Chitwood, D. D., McCoy, H. V., McCoy, C. B., Ludwig, D. D., \& Edlin, B. R. (1992). Crack cocaine use and sexual activity in Miami, Florida. J Psychoactive Drugs, 24(4), 373-380. https://doi.org/10.1080/02791072.1992.10471661

Wechsberg, W. M., Luseno, W. K., \& Lam, W. K. (2005). Violence against substance-abusing South African sex workers: intersection with culture and HIV risk. AIDS Care, 17(Suppl 1), S55-64. https://doi.org/10.1080/09540120500120419

Wei, S. B., Chen, Z. D., Zhou, W., Wu, F. B., Li, S. P., \& Shan, J. G. (2004). A study of commercial sex and HIV/STI-related risk factors among hospitality girls in entertainment establishments in Wuhan, China. Sex Health, 1(3), 141-144. https://doi.org/10.1071/SH03011

Whitaker, D. J., \& Miller, K. S. (2000). Parent-adolescent discussions about sex and condoms impact on peer influences of sexual risk behavior. Journal of Adolescent Research, 15(2), 251-273. https://doi.org/10.1177/0743558400152004

Wojcicki, J. M., \& Malala, J. (2001). Condom use, power and HIV/AIDS risk: sex-workers bargain for survival in Hillbrow/Joubert Park/Berea, Johannesburg. Soc Sci Med, 53(1), 99-121. 
https://doi.org/10.1016/S0277-9536(00)00315-4

Xu, J. J., Smith, M. K., Chu, J., Ding, G. W., Chang, D. F., Sharp, G. B., ... \& Wang, N. (2012). Dynamics of the HIV epidemic in southern China: sexual and drug-using behaviours among female sex workers and male clients in Yunnan. International journal of STD \& AIDS, 23(9), 670-675. https://doi.org/10.1258/ijsa.2009.009128

Xu, J. J., Wang, N., Lu, L., Pu, Y., Zhang, G. L., Wong, M., ... \& Zheng, X. W. (2008). HIV and STIs in clients and female sex workers in mining regions of Gejiu City, China. Sexually transmitted diseases, 35(6), 558-565. https://doi.org/10.1097/OLQ.0b013e318165926b

Yang, H., Li, X., Stanton, B., Liu, H., Liu, H., Wang, N., ... \& Chen, X. (2005). Heterosexual transmission of HIV in China: A systematic review of behavioral studies in the past two decades. Sexually transmitted diseases, 32(5), 270. https://doi.org/10.1097/01.olq.0000162360.11910.5a

Yu, Y. J. (2013). Subjectivity, hygiene, and STI prevention: a normalization paradox in the cleanliness practices of female sex workers in post-socialist China. Med Anthropol Q, 27(3), 348-367. https://doi.org/10.1111/maq.12050

Zhang, C., Li, X., Hong, Y., Zhou, Y., Liu, W., \& Stanton, B. (2013). Unprotected sex with their clients among low-paying female sex workers in southwest China. AIDS Care, 25(4), 503-506. https://doi.org/10.1080/09540121.2012.726345

\section{Copyrights}

Copyright for this article is retained by the author(s), with first publication rights granted to the journal.

This is an open-access article distributed under the terms and conditions of the Creative Commons Attribution license (http://creativecommons.org/licenses/by/4.0/). 